\title{
La arquitectura del pastoreo: Uso del espacio y sistema de asentamientos en la Puna de Atacama (Susques)
}

BARBARA GÖBEL ${ }^{1}$

\section{RESUMEN}

El objetivo del trabajo es contribuir al conocimiento de la arquitectura del pastoreo en los Andes. El foco regional del estudio es Huancar en el Departamento Susques (Jujuy, Argentina). En primer lugar se describen los sistemas de asentamientos vinculados con las actividades pastoriles de Huancar y luego se presentan los esquemas del uso del espacio por parte de los pastores. Finalmente, se analizan los significados que el espacio tiene para los pastores.

Palabras claves: Puna de Atacama - pastoreo - sistema se asentamientos - uso del espacio.

\section{ABSTRACT}

This paper aims to contribute to the existing knowledge on pastoralist architecture in the Andes. The regional focus of the study is put on Huancar, Department of Susques (Jujuy, Argentina). We describe first the settlement structure related to the pastoralist activities of Huancar; then, we present the pastoralists' spatial patterns of use. Finally, we analyze the cultural meanings that space has for pastoralists.

Key words: Puna de Atacama - pastoralism - settlement structure - spatial patterns.

Recibido: marzo 2002. Manuscrito revisado aceptado: agosto 2003.

\section{Introducción}

El pastoreo es la única estrategia productiva viable en regiones áridas como las tierras altoandinas, en las que faltan mayores concentraciones de pasturas y en las que el cultivo extensivo de plantas ya no es posible. La movilidad es su característica más llamativa. A diferencia de la agricultura, el pastoreo es una actividad económica, en la cual los medios de producción no son fijos, sino que caminan sobre patas. Los animales son

1 Institut für Altamerikanistik und Ethnologie, Universität Bonn, Römerstr. 164, D-53117 Bonn. Email: bgoebel@unibonn.de; International Human Dimensions Programme on Global Environmental Change (IHDP), Walter-Flex-Str. 3, D-53117 Bonn. Email: goebel.ihdp@uni-bonn.de “despensas ambulantes" (Clutton-Brock 1989). Al no existir pasturas cercadas, en las que puede ser encerrado el ganado, los movimientos de los animales tienen que ser controlados por los hombres. Ellos se trasladan con el ganado de un área de pastoreo a otra y dirigen también allí sus desplazamientos. Por lo tanto, el pastoreo no implica solamente una relación particular con los animales, sino que también con el espacio.

La relación hombre-espacio en el contexto de economías móviles ha sido estudiada básicamente para sociedades de cazadores-recolectores. Especialmente los trabajos sobre los sistemas de asentamientos vinculados con la caza y la recolección han marcado nuestras ideas sobre la visibilidad material de un estilo de vida móvil (ver p.e., Biesele et al. 1999; Ingold et al. 1988; Kelly 1995; Lee y De Vore 1968; Lee y Daly 1999; Winterhalder y Smith 1981). Han sido de gran relevancia heurística no solamente para la antropología social sino también para la arqueología. Existen bastante menos estudios sobre los sistemas de asentamientos vinculados con el pastoreo. Además, la mayoría de ellos sólo se basa en estudios de caso africanos y asiáticos (Cribb 1991; Dyson-Hudson y Dyson-Hudson 1980; Fratkin et al. 1994; Fratkin 1997).

La falta de información sistemática sobre la arquitectura del pastoreo en los Andes está relacionada con la posición marginal que el pastoreo andino tiene en la antropología en general. Mientras que los sistemas pastoriles africanos y asiáticos influenciaron a importantes desarrollos teóricos (ver p.e., Barfield 1993; Dyson-Hudson y Dyson-Hudson 1980; Galaty y Johnson 1990; Khazanov 1994; Salzman y Galaty 1990), el análisis del pastoreo andino muy pocas veces ha trascendido el marco de la antropología regional. Los primeros antropólogos sociales, que a principios de los años sesenta describieron a poblaciones andinas, cuya subsistencia se basa en la cría móvil de ganado, fueron Jorge Flores Ochoa (1968), 
Glynn Custred (1977) y Horst Nachtigall (1966). Sus estudios inspiraron a una serie de trabajos sobre la producción pastoril en los Andes, el intercambio con agricultores, la organización social de los pastores y su vida religiosa. ${ }^{2}$ La mayoría de las investigaciones se llevaron a cabo en el altiplano peruano y boliviano. Poseemos mucho menos información etnográfica sobre el norte de Chile y el Noroeste Argentino.

El presente trabajo se basa en una investigación realizada en uno de los sectores de los Andes poco estudiados hasta ahora: la región de Susques en el Noroeste Argentino; más precisamente el Distrito de Huancar. Presentaremos algunos resultados de nuestro análisis de la arquitectura del pastoreo en esta región. En primer lugar, describiremos las estructuras arquitectónicas y los lugares de uso específico para actividades pastoriles en Huancar. ${ }^{3}$ Después analizaremos con qué esquemas de uso del espacio están vinculados estas estructuras arquitectónicas y estos lugares. También discutiremos los signficados que el espacio puede tener para los pastores. Como este trabajo no puede ser más que un primer aporte hacia una comprensión más amplia de la arquitectura del pastoreo en los Andes, priorizamos en él la presentación de información etnográfica.

2 Para compilaciones y resúmenes del estado de la investigación véanse Bonavía (1996), Browman (1974, 1989), Flores Ochoa (1977, 1983, 1988), Flores Ochoa y Kobayashi (2000), Orlove (1981), Palacios Ríos (1987), Webster (1973).

3 La información etnográfica e histórica que presentamos en este trabajo fue recopilada durante varias estadías en Huancar (años 1991-93, 1994, 1995, 1996-97, 1999, 2000). Estas estadías se enmarcaron en diferentes proyectos de investigación. En un primer proyecto estudiamos el pastoreo, el intercambio económico y la organización social de estas actividades (y sus transformaciones a lo largo del tiempo). Nos interesamos particularmente por el manejo de riesgos e inseguridades por parte de los habitantes de este sector tan árido de las tierras altoandinas. En un segundo proyecto analizamos las complejas vinculaciones entre percepción ambiental, cosmovisión y aprovechamiento de recursos. Uno de nuestros focos de análisis fueron las relaciones hombre-animal. El tercer proyecto consistió en una comparación intercultural de diferentes conocimientos ambientales, percepciones de riesgo y percepciones de uso y de control del medio ambiente (p.e., pastores, maestros, ingenieros agrónomos).

\section{Estructuras socioeconómicas del área de estudio: El Distrito de Huancar}

El Distrito de Huancar pertenece al Departamento de Susques de la Provincia de Jujuy (Figura 1). Se ubica en el borde oriental de la Puna de Atacama, que es uno de los sectores más áridos de las tierras altoandinas (Troll 1968). El sector oriental de la Puna de Atacama es un semidesierto de altura, caracterizado por la presencia de salares y una vegetación escasa y dispersa, que consiste básicamente en pequeños arbustos (tolares) y gramíneas. A diferencia de otros sectores de las tierras altoandinas no existe en todo el Distrito de Huancar una "vega" o un "bofedal". La mayoría de las fuentes de agua no son permanentes y se encuentran esparcidas en las quebradas con grandes distancias entre sí.

Para la mayoría de los 320 habitantes de Huancar la cría de ganado es de gran importancia económica y cultural. A mediados de 1993 el $83 \%$ de las 46 unidades domésticas se autodeterminaban como "familias que pastorean hacienda"; en 1996 fue el $80 \%$ (36 de un total de 45 unidades domésticas) y en 1999 fue el $70 \%$ (36 de un total de 51 unidades domésticas). Los rebaños de las familias de Huancar están compuestos por llamas, ovejas y cabras. El conjunto de estos animales es denominado "hacienda". Existe una relación afectiva muy estrecha con la hacienda; llamas, ovejas y cabras son percibidos como miembros no-humanos de la unidad doméstica. La hacienda de una familia abarca en promedio unos 150 animales, siendo el rango de 35 a 475 animales, según los censos ganaderos (1986-1999) que pudimos recopilar. Cuatro familias tienen 300 o más animales, mientras que el tamaño de los rebaños de otras dos familias sólo ronda las 50 cabezas. Un rebaño de 50 animales es considerado el mínimo para que puedan subistir dos personas adultas de la cría del ganado. La movilidad espacial con los rebaños es bastante alta. Aparte de la hacienda, cada familia posee al menos media docena de burros; algunas tienen además una o dos mulas. Estos animales no son pastoreados, por lo que corren libremente por los cerros. ${ }^{4}$

A diferencia de otras regiones de los Andes, no existe en Huancar una estructura de comunidad

4 Para más información sobre la producción pastoril en 


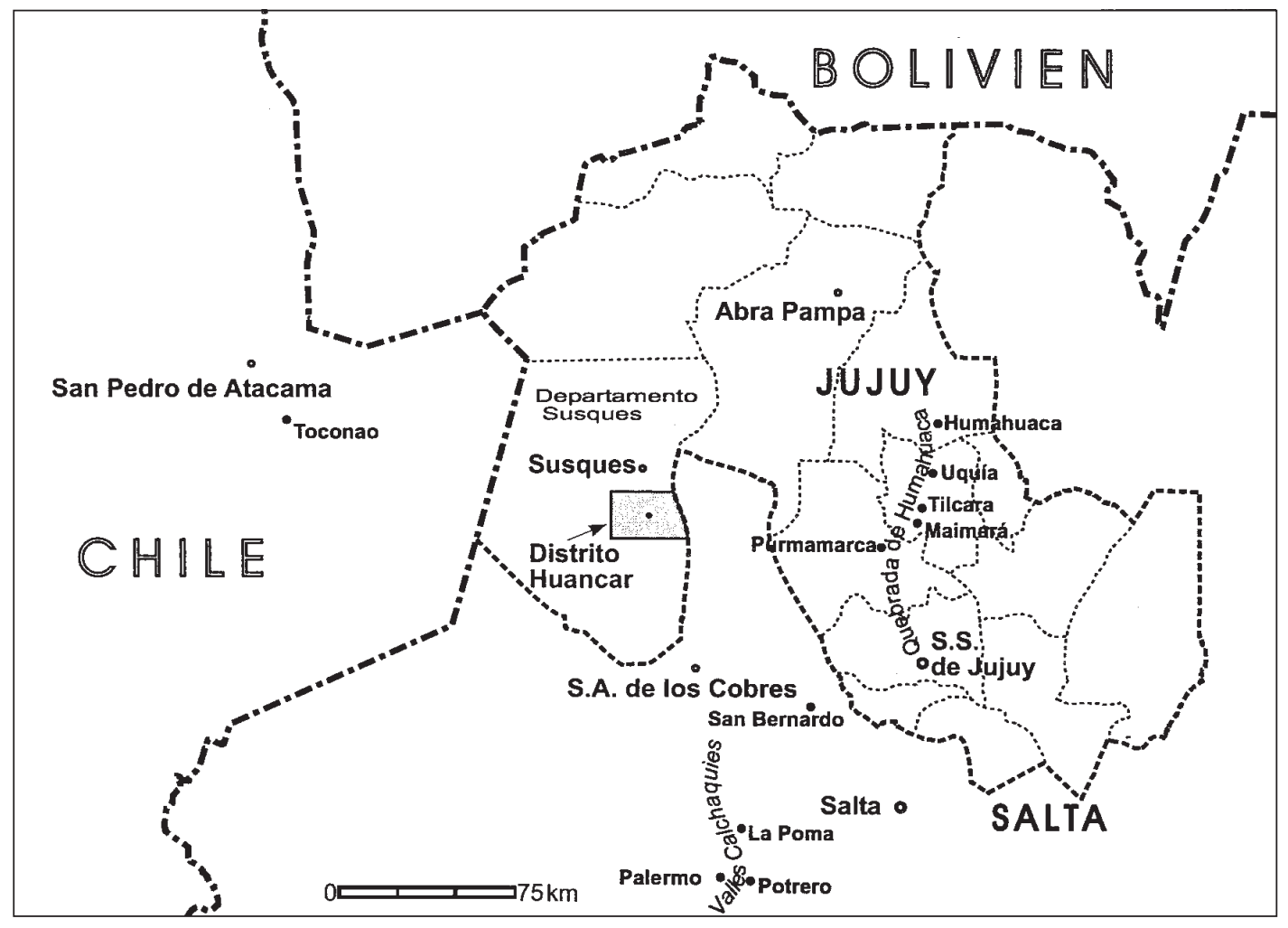

Figura 1. Ubicación del Distrito de Huancar en la Provincia de Jujuy.

muy desarrollada. Faltan instituciones comunitarias como ayllus, sistemas de cargos religiosos o el uso conjunto de tierras y recursos. Tampoco existe en Huancar una identidad étnica explicitada. Los pobladores se definen como "gentis de acá". No se autodeterminan "atacameños", ni tampoco adoptaron adscripciones étnicas externas como el nombre de kollas (Göbel, en prensa b). Relacionado con la falta de una estructura de comunidad desarrollada no encontramos en Huancar un sistema coherente y abarcativo de ejes espaciales, que defina un territorio comunitario con fronteras claras. No encontramos, por ejemplo, un sistema de cerros o lugares sobresalientes, que compartan todos los pobladores de Huancar como puntos de referencia centrales para su identidad. ${ }^{5}$

5 Astvaldsson (1997), Bastien (1978), Bolin (1998), Dransart (2002), Gose (1994), Isbell (1978), Martínez (1976), Valderrama y Escalante (1988) y Wachtel (1990) describen tales sistemas de cerros o lugares sobresalientes -mallkus, uywiris o apus, wak'as, etc.- y analizan su relevancia para procesos identidarios.
El eje central de la organización social y económica del espacio en Huancar no es la comunidad, sino la unidad doméstica. Las unidades domésticas son denominadas "familias". Son concebidas como las arenas centrales de producción, de distribución, de consumo y de reproducción social. Abarcan en promedio unas siete personas. $\mathrm{La}$ mayoría de ellas está compuesta de tres generaciones (padres o madre, hijos e hijas, hijos de las hijas).

Cada unidad doméstica usufructa determinadas tierras de pastoreo y fuentes de agua para los hombres y el ganado. ${ }^{6} \mathrm{O}$ sea que, a diferencia de sec-

6 Legalmente las tierras siguen siendo de propiedad fiscal, o sea pertenecen al Estado provincial. Los pobladores de Huancar intentan desde algunos años obtener los títulos de sus tierras advocándose a la nueva legislación indígena que estableció la reforma de la Constitución Argentina en 1994. La actual legislación indígena permite a comunidades aborígenes que se han inscrito con personería jurídica en el Instituto Nacional de Asuntos Indígenas (INAI) obtener títulos individuales o comunitarios de las tierras que habitan 
tores más húmedos de las tierras altoandinas, que presentan concentraciones de recursos forrajeros como bofedales o vegas, no existen en Huancar pastoreos o aguadas comunitarias. ${ }^{7}$ La historia oral constata que esto no es un desarrollo reciente, sino que ha sido una constante en Huancar. Los pastoreos de cada familia tienen en promedio unas 1.649 ha, abarcando el espectro de 115 ha (unidad doméstica 47) a más de 11.000 ha (unidad doméstica 22; ver Figura 2). El derecho exclusivo de uso de las unidades domésticas no se extiende, sin embargo, a la extracción de leña o al pastar de burros y mulas. Los burros y las mulas no son pastoreados y corren dispersos por los cerros sin mayor control. ${ }^{8}$

Debido a la centralidad de las unidades domésticas en Huancar, la identidad local es mucho más fuerte que la identidad comunitaria. El sentimiento de arraigo local, es decir, el compromiso que una persona siente hacia el espacio que su familia controla (tanto económica como simbólicamente) y que socialmente existe adscrito a ella, es mucho más grande que su sentido de pertenencia a una comunidad abarcativa.

\section{Las unidades domésticas como ejes de la organización social y económica del espacio}

El sistema de asentamientos vinculado a las actividades pastoriles en Huancar tiene tres componentes: a) la casa central o "casa de campo"; b) los puestos temporarios o "estancias", distribuidos en el área de pastoreo de la unidad doméstica, y c) la "casa en el pueblo", que se encuentra en el pequeño pueblo de Huancar en el centro del distrito. Esta combinación de una base central con puestos temporarios y eventualmente una casa en el centro administrativo y comercial más cercano es bastante común en los siste-

tradicionalmente. Los pobladores de Huancar se han constituido a fines de los '90 como una comunidad aborígen, obteniendo hace poco la personería jurídica de la misma. Mientras que oficialmente piden los títulos comunitarios de sus tierras (pastoreos y aguadas), internamente ya han decidido que las familias seguirán teniendo derechos exclusivos sobre las tierras de pastoreo y las aguadas.

7 Compárese con Brush y Guillet (1985: 26), Browman (1974 y 1989), Orlove (1981) y West (1983: 94).

8 Se puede extraer leña (tola, checal, añagua) en las tierras de pastoreo de otras familias, siempre y cuando no sea alrededor de la casa de campo, los puestos o los corrales. mas pastoriles andinos (ver p.e., Browman 1989; Brush 1977; Brush y Guillet 1985; Cárdenas 1990; Flannery et al. 1989; Flores Ochoa 1968, 1977 y 1988; Gundermann 1984; McCorkle 1987; Nachtigall 1966; Orlove 1981). Sin embargo, difieren los nombres que reciben los componentes en las diferentes partes de los Andes. Así, en muchos lugares "estancia" no es la denominación de un puesto temporario, sino de una casa central o de un caserío con las casas centrales de varias familias (ver p.e., Caro 1985; Dransart 2002; Flannery et al. 1989; Flores Ochoa 1968; Greslou 1989; Inamura 1981; Martínez 1975; McCorkle 1987; Nabeshima 2000; Orlove 1981). ${ }^{9}$

\section{La "casa de campo"}

En el centro del espacio controlado por una familia se encuentra la "casa de campo". Los miembros de una unidad doméstica son identificados por las otras familias de la región con el nombre del lugar, en el que se encuentra su "casa de campo": Barrancas, Agua Buena, Cuevas, etc. (Figuras 3 y 4). El nombre del lugar de la "casa de campo" es el lugar que una persona menciona, cuando se le pregunta por su origen y por su pertenencia. Es un icono, que condensa todo un sistema de ocupación espacial, derechos de uso de recursos y de prácticas económicas. Además, la "casa de campo" constituye para los miembros de una unidad doméstica una referencia clave cuando evocan relaciones sociales actuales y pasadas. Así, por ejemplo, las genealogías son narradas y memorizadas con la ayuda de los nombres de los lugares de "casa de campo". En la "casa de campo" se llevan también a cabo los rituales que activan y refuerzan las relaciones de los miembros de una familia con la pachamama (p.e., la señalada, $1^{\circ}$ de agosto, días de los santos de los animales). Las relaciones con la pachamama son esenciales para mantener el bienestar de las

9 Gundermann (1984: 111) define, en su trabajo sobre los aymaras de Isluga, "estancias" como "unidades territoriales pertenecientes en mancomún a coaliciones de familias reclutadas por vía patrilineal". Denominaciones comunes para puestos temporarios son kancha (Flannery et al. 1989), “cabaña” (Flores Ochoa 1968; Cárdenas 1990; Greslou 1989) o paskana (Gundermann 1984: 113). Para una discusión general, ver también Nielsen (2001). 


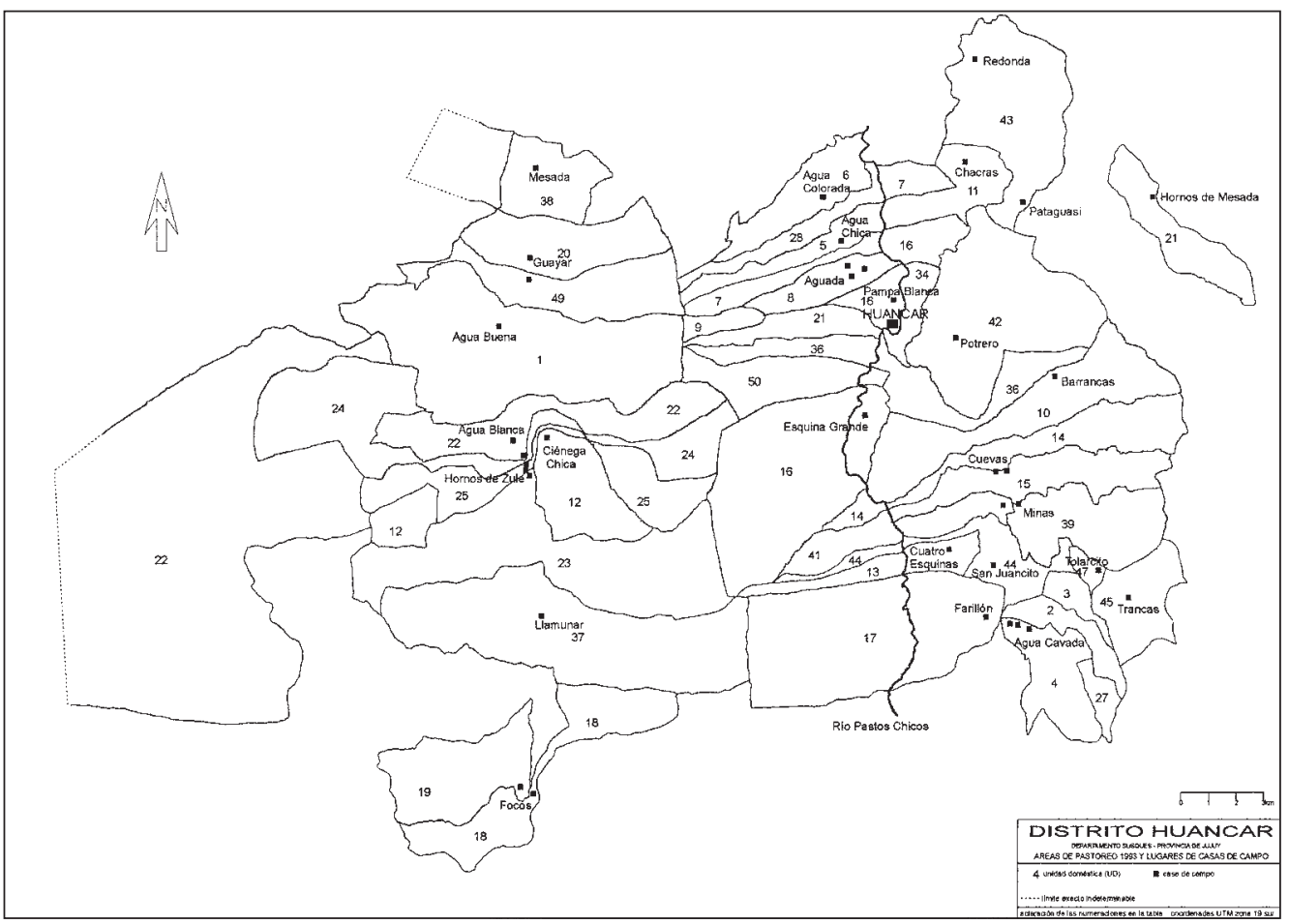

Figura 2. Distrito de Huancar: Áreas de pastoreo con lugares de casas de campo.

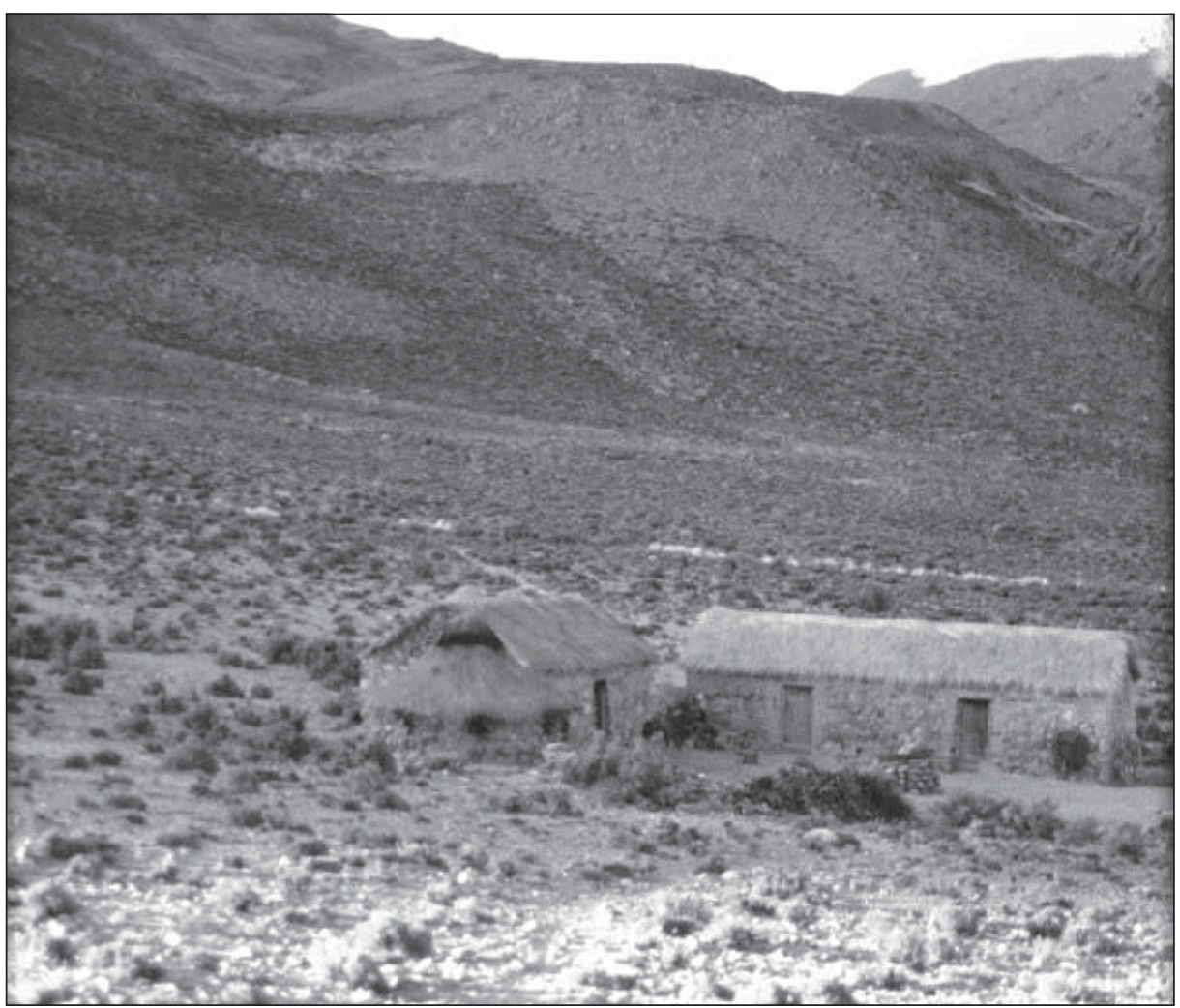

Figura 3. Casa de campo Agua Buena (unidad doméstica 1). 


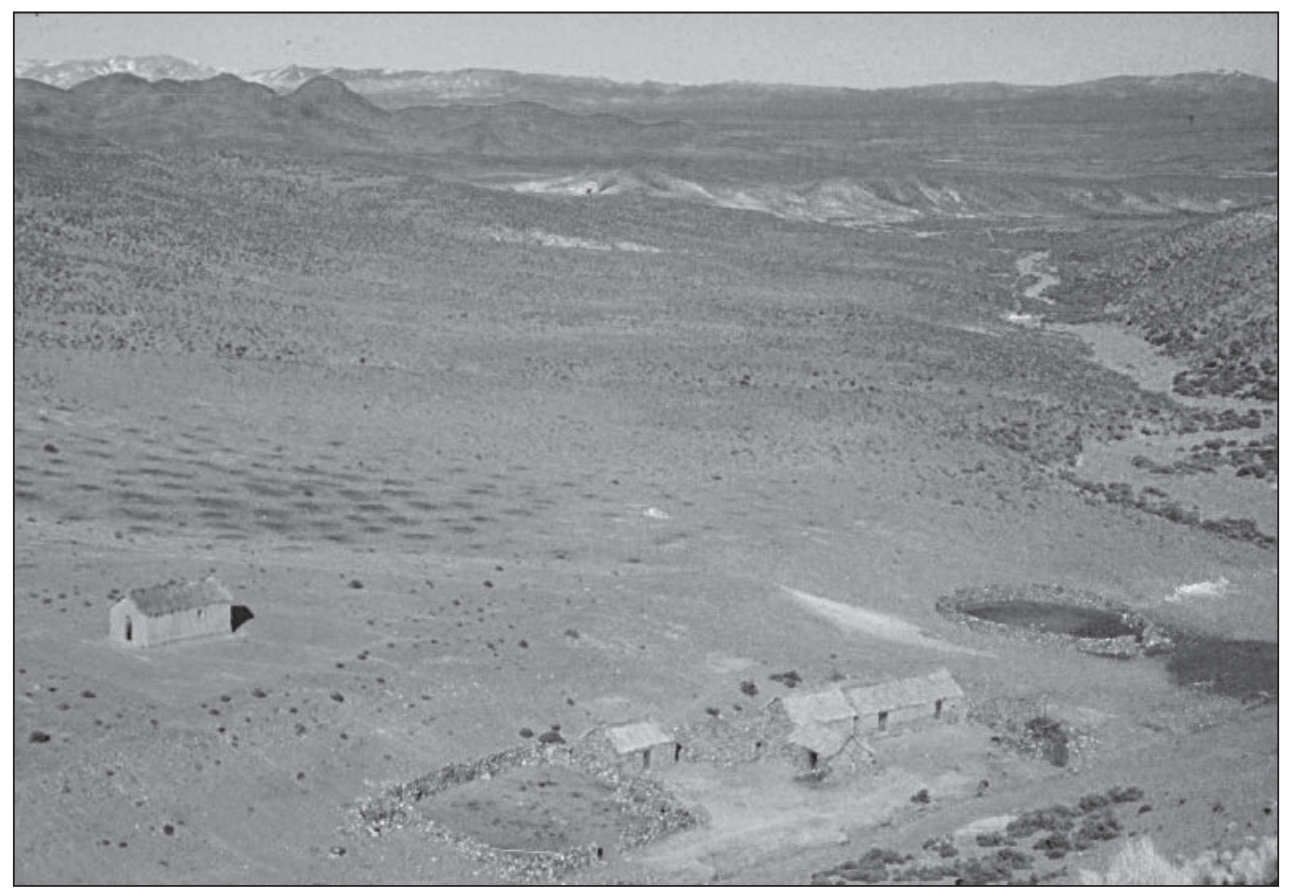

Figura 4. Casa de campo Barrancas (unidad doméstica 10).

personas y del ganado y, por lo tanto, asegurar la producción pastoril (Göbel, en prensa c). La "casa de campo" es, por lo tanto, el principal archivo material de las prácticas económicas, sociales y religiosas de una familia.

\section{Elementos típicos de una casa de campo. Una} "casa de campo" no consiste solamente en una casa. Más bien es todo un complejo de estructuras, instalaciones y áreas de uso específico. Hay que aclarar que en los Andes una casa tradicionalmente sólo contiene un cuarto y no varios cuartos. ${ }^{10}$ Las casas tienen planta rectangular, están hechas de piedra con techos a dos aguas cubiertos de guaya (paja). No poseen ventanas y sólo tienen una puerta, que en general es de madera de cardón.

10 También Boman (1908: 429), quien reccorrió en 1903 el sector oriental de la Puna de Atacama, escribe: "La maison n'a qu'une seule chambre". López Aranda (1937: 39) constata en su informe sobre el territorio de los Andes: "Viven en pequeñas casas que, por lo general, constan de una habitación". Y Von Rosen (1957: 77), quien exploró en 1901 la Puna de Jujuy y el borde de la Puna de Atacama, señala: "Viven en pequeños pueblos o en casas y ranchos solitarios y alejados. Sus habitaciones ... [c]onstan de una sola pieza".
Estos son los elementos típicos de una "casa de campo" (Figura 5, para los elementos 1-10, 12-14, 16-18): ${ }^{11}$

1) Una a dos "casas de familia". Ellas funcionan básicamente como dormitorios de los miembros de la unidad doméstica.

2) La casa de huéspedes ("ramada"). En ella duerme la visita. Cuando vienen varias personas de visita, ellas comen en la "ramada" y no con la familia en la kancha. Los miembros de una unidad doméstica mantienen siempre cierta distancia hacia la visita, independientemente si son parientes, amigos o vecinos que los visitan. También se realizan en la "ramada" fiestas y se llevan a cabo rituales (primer corte de pelo, señalada, $1^{\circ}$ de Agosto, carnaval y rodeo de los burros, etc.).

3) El "depósito", para guardar mercadería, carne, vellones de lana, herramientas, etc.

11 Para otros croquis de "casas de campo" pastoriles ver Yacobaccio y colaboradores (1998: 39, 41) para Susques (Jujuy, Argentina), y Flannery y colaboradores (1989: 52, 57, 73) para Ayacucho (Perú). Lamentablemente este último no presenta escala. 


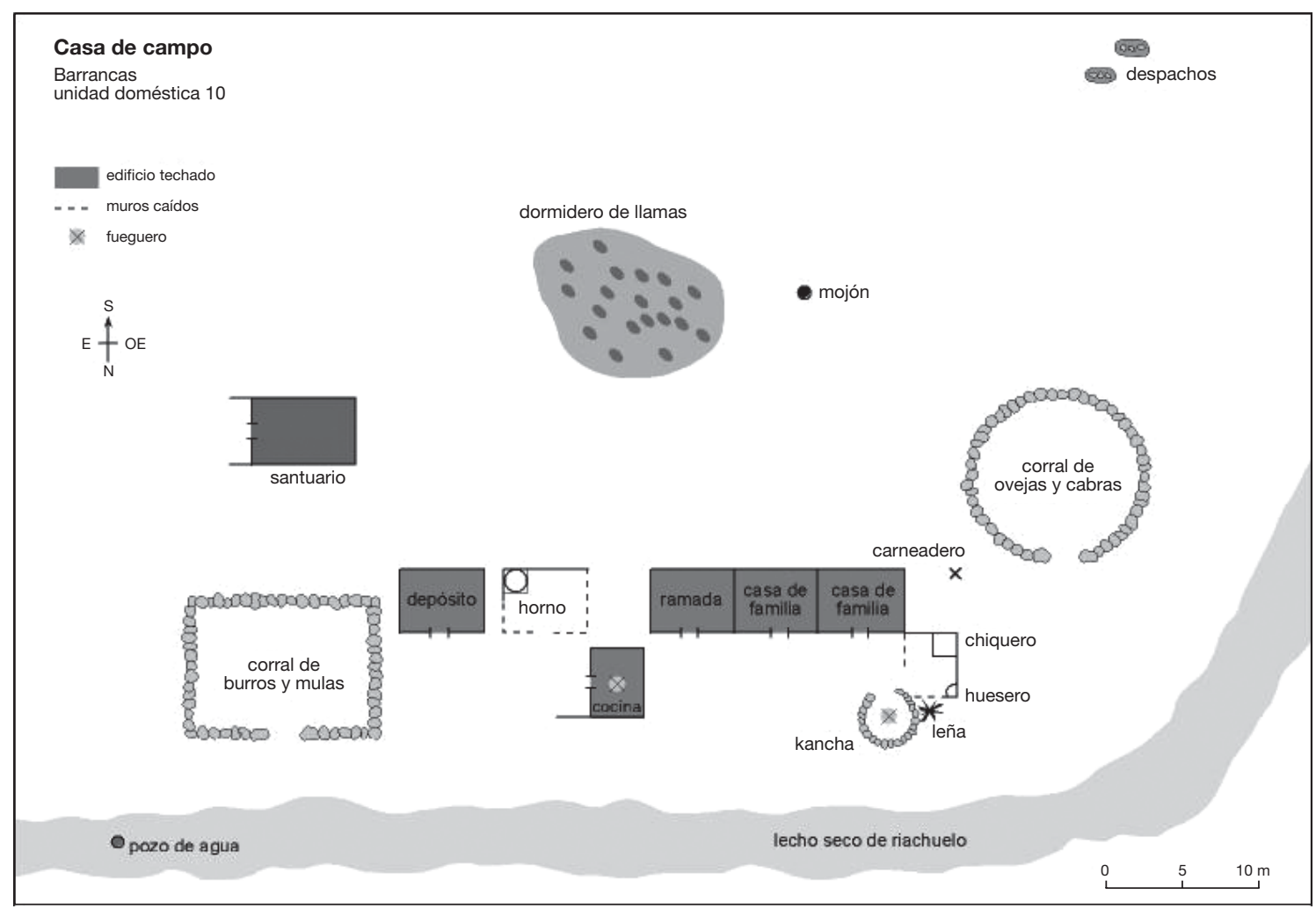

Figura 5. Croquis de la casa de campo Barrancas (unidad doméstica 10).

4) La cocina para uso diario, la kancha. En general, ella sólo consiste en un semicírculo de piedras o arbustos (tolas) de alrededor de $1 \mathrm{~m}$ de altura con un fogón (fueguero) en el centro. Kancha significa (en quechua) corral. $^{12}$ "La kancha es el corral para la gente", dicen los habitantes de Huancar. El paralelismo entre el "corral de la gente" y el "corral de la hacienda" se hace más evidente todavía en los puestos temporarios, donde la kancha funciona también como dormitorio.

5) La "cocina techada" para ocasiones excepcionales como la realización de rituales (señala$d a, 1^{\circ}$ de Agosto, días de los santos de los animales, etc.) y de fiestas (carnaval, rodeo de los burros, etc.). En las "casas de campo" ubicadas al oeste del río Pastos Chicos, o sea en el doblado (quebrada transversal), la cocina tiene generalmente una planta circular y

12 En Isluga (norte de Chile), por ejemplo, los corrales de los animales reciben el nombre de kancha (Dransart 2002: 84). un techo cónico (ver Figura 3). En las "casas de campo" ubicadas hacia el este del río, o sea en la banda, la cocina tiene generalmente una planta rectangular o cuadrada y un techo a dos aguas (ver Figuras 4 y 5 y Figura 2 para la ubicación de la unidad doméstica 1 en el doblado y la unidad doméstica 10 en la banda). En el centro de la cocina se encuentra un fogón (fueguero).

6) El "horno" para hacer pan. También se utiliza al "horno" para cocinar la cabeza de llama, oveja o cabra ("cabeza guateada"), lo que es considerado como una comida para ocasiones especiales.

7) El "corral para ovejas". Es circular y está hecho de piedras o arbustos (tolas). En él se encierran ovejas y cabras.

8) El "corral para burros y mulas". En general este corral es rectangular. También es utilizado para esquilar, capar o curar a las llamas.

9) Un área, en la que se echan las llamas al atardecer para dormir: el "dormidero". Se le reconoce por las manchas de depósitos fecales de 
las llamas. ${ }^{13}$ Las llamas, que a diferencia de las ovejas y las cabras no comen durante la noche, llegan al atardecer al "dormidero" y se levantan solas en la madrugada para salir a pastar.

10) El "chiquero": da es un pequeño corralito de piedra, en el que se encierran los corderitos y los chivitos más chicos y débiles, cuando las pastoras salen durante el día con el ganado.

11) El "pozo para las guaguas". Este es un agujero de unos $30 \mathrm{~cm}$ de diámetro y $40 \mathrm{~cm}$ de profundidad que se encuentra cerca de las casas. Funciona como un corralito. En él se paran los niños de uno a dos años de edad.

12) El "huesero". Es un depósito cilíndrico de piedra que funciona como basurero de los huesos usados y desechados. Existe la norma de que no se deben desparramar los huesos después de la comida. La pachamama interpretaría esto como una falta de respeto frente al animal que fue sacrificado para saciar el hambre de los hombres. En consecuencia, castigaría a toda la unidad doméstica.

13) El "carneadero". Solamente durante los rituales se puede matar a un animal dentro del corral. Los pastores justifican esta prohibición con el respeto que los humanos deben mantener hacia la "casa" de la "hacienda" y los riesgos involucrados en la matanza de un animal para el uso humano (Göbel, en prensa c). En contextos cotidianos los animales son sacrificados fuera del corral, debajo de un arbusto que se encuentra a unos $50 \mathrm{~m}$ del corral. Se reconoce al "carneadero" por el contenido verde-marrón de las tripas (pusno) que es exprimido sobre el arbusto y por las piedras blancas que se encuentran debajo del arbusto. Estas piedras tapan el agujero en el que se realizan ofrendas de alcohol y hojas de coca (ch'allas) a la pachamama, antes de degollar al animal. Tales ch'allas acompañan a cualquier intervención de cierta importancia con el animal (sacrificio, esquila, capa, etc.). Es importante pedirle permiso a la pachamama antes de hacer algo con

13 Tantos las especies domésticas de los camélidos -la llama (Lama glama) y la alpaca (Lama pacos) - como las especies silvestres -el guanaco (Lama guanacoe) y la vicuña (Lama vicugna)- depositan sus excrementos en los lugares, en los que duermen durante la noche. Las almohadas de guano, sobre las que se acuestan los animales, son muy buenos termoaislantes. Por lo tanto, protegen a los camélidos de las peligrosas heladas nocturnas en las tierras altas (Troll 1980: 35). un animal, para no poner en peligro el bienestar de toda la "hacienda" y por ende la producción pastoril (Göbel, en prensa c).

14) Una fuente de agua, ya sea manantial ("ojo de agua") o pozo cavado en el lecho seco de un riachuelo. De esta fuente de agua se saca con una lata el agua para beber y cocinar, para el aseo personal y el lavado de vajilla y ropa. Los animales toman agua en otra fuente de agua en la cercanía de la casa de campo.

15) Algunas "casas de campo" tienen también un pequeño "rastrojo" y un telar. Tanto el cultivo de plantas como el trabajo en telar son actividades masculinas, por lo que su existencia en una "casa de campo" depende de la presencia de un hombre adulto en la unidad doméstica. En el rastrojo los hombres cultivan verdura, papa, quinoa o alfalfa. Con el telar español ellos tejen mantas, frazadas y barracanes para el uso doméstico o el intercambio económico.

16) Todas las "casas de campo" tienen un altar con los "santos de la hacienda" y los "santos de la gente". El altar se puede encontrar en un edificio especial de planta rectangular, un "santuario", o en un sector de la "casa de familia" ¿Cuáles son los santos del ganado? El santo de las llamas es San Antonio (13 de junio), el de las ovejas San Juan (24 de junio), el de las cabras es San Bartolo (24 de agosto) y el de los burros es San Ramón (31 de agosto). Muchas familias tienen además una imagen de la Virgen del Perpetuo Socorro (primer domingo después de San Juan) o de la Virgen de los Remedios ( $1^{\circ}$ de septiembre) en su altar. En el día de estos santos se realizan rituales en la "casa de campo".

17) El "mojón". Este es un montículo cónico de piedras blancas, que se encuentra cerca del corral de ovejas y cabras (a una distancia de aproximadamente $50 \mathrm{~m}$ ). Las piedras blancas representan a las llamas, las ovejas y las cabras de una familia; o sea simbolizan la reproducción del ganado. El "mojón" juega un rol central en la señalada, que es el ritual más importante del ciclo productivo ganadero.

18) Los "despachos". Son tumbas que están ubicadas a una distancia entre $250 \mathrm{~m}$ y $500 \mathrm{~m}$ de la "casa de campo". Se trata de unos pequeños montículos rectangulares de tierra con una hilera de piedra en la parte más alta. En los "despachos" se entierra, nueve días después de la muerte de un miembro de la familia, a un pequeño muñeco, que representa a la per- 
sona fallecida. La gente de Huancar cree que no alcanza con enterrar el cuerpo del familiar muerto en el cementerio cercano al pueblo. También hay que enterrar el alma del familiar muerto cerca de su lugar de pertenencia: la "casa de campo". Esto subraya la centralidad del sentimiento de arraigo local. Y también muestra la preocupación por mantener la integridad del espacio familiar, tratando de prevenir la disolución de los vínculos entre personas y espacios con sus diversas representaciones materiales.

Es importante señalar que la "casa de campo" siempre es percibida como todo el conjunto de las estructuras materiales que hemos enumerado. Es todo un complejo de edificios, instalaciones y lugares de uso específico que abarca desde viviendas para las personas ("casas de familia", "ramada", depósito, cocina, kancha), el ganado (corrales y dormidero), los santos (santuario) y los muertos ("despachos") hasta estructuras menos visibles asociadas con las prácticas pastoriles como el "huesero", el pozo de agua o el carneadero.

Semejanzas y diferencias entre "casas de campo". En la Tabla 1 resumimos la distribución de algunas de las estructuras descritas en las 42 "casas de campo" que documentamos en los años 1992/93. El relevamiento incluye también a algunas "casas de campo" de familias que migraron (unidades domésticas 49, 50) y de familias que ya no crían ganado (unidades domésticas 27, 28).

\begin{tabular}{|lcc|}
\hline Estructura & Casas de campo & $(\%)$ \\
\hline casa(s) & 42 & 100 \\
corral(es) & 42 & 100 \\
kancha & 42 & 100 \\
carneadero & 42 & 100 \\
huesero & 42 & 100 \\
cormidero & 42 & 100 \\
mojón & 42 & 100 \\
despacho & 42 & 100 \\
fuente de agua & 42 & 100 \\
cocina techada & 32 & 76 \\
horno & 25 & 59.5 \\
telar & 24 & 57 \\
rastrojo & 15 & 38 \\
santuario & 6 & 14 \\
letrina & 4 & 9.5 \\
\hline
\end{tabular}

Tabla 1. Distribución de estructuras típicas en las casas de campo (1992/93).
Esto explica por qué el número total de "casas de campo" es mayor que el de las unidades domésticas pastoriles (38 en 1993). En la taxonomía local el término "casas" abarca "casas de familia", "ramadas" y depósitos. En cambio la "cocina techada" no es categorizada como una "casa".

Vemos entonces que en todas las "casas de campo" aparece un núcleo de elementos -casa, corral, kancha, carneadero, huesero, dormidero, mojón, despacho, fuente de agua-, mientras que la distribución de otros elementos varía. Además, existen diferencias con respecto a la cantidad de casas y corrales que tiene cada "casa de campo". Hemos resumido estas diferencias en la Tabla 2.

Cuanto menos cantidad de casas tiene una unidad doméstica, tanto más funciones tienen esas casas (compárense Figuras 3 y 4). Si, por ejemplo, una unidad doméstica sólo tiene una casa, esta funciona también como "ramada" cuando llega visita. Si pernocta la visita en el lugar, los dueños de casa duermen en la kancha. Si la familia no posee un santuario, un pequeño altar con los "santos de la hacienda" y los "santos de la gente" estará ubicado en una parte no muy expuesta de la casa. Todas las unidades domésticas tienen un corral para las ovejas y las cabras. Si tienen un segundo corral, este es para los burros y las mulas. Las pocas unidades domésticas con tres corrales destinan uno de ellos a las llamas. Esto no significa necesariamente, que encierran allí a las llamas durante la noche. Más bien utilizan este "corral para las llamas" en actividades extraordinarias con ellas como la esquila, la capa, la curación y en los rituales.

El trabajo etnoarqueológico de Yacobaccio, Madero y Malmierca (1998) en las cercanías del pueblo de Susques confirma a grandes rasgos la com-

\begin{tabular}{|lcc|}
\hline Estructura & Casas de campo & $(\%)$ \\
\hline 2 casas & 14 & 33.5 \\
1 casa & 13 & 31 \\
4 casas & 8 & 19 \\
3 casas & 5 & 12 \\
5 casas & 2 & 4.5 \\
1 corral & 23 & 55 \\
2 corrales & 17 & 40.5 \\
3 corrales & 2 & 4.5 \\
\hline
\end{tabular}

Tabla 2. Cantidad de casas y corrales de las casas de campo (1992/93). 
posición de "casas de campo" que hemos presentado aquí. Los autores también hacen hincapié en la variabilidad que puede existir entre una "casa de campo" grande y una más pequeña. En su comparación de las bases residenciales ("casas de campo" ${ }^{14}$ de dos unidades domésticas pastoriles, los autores describen una serie de los elementos característicos de una "casa de campo", que pudimos determinar en nuestros estudios en Huancar. La base residencial más grande, Lapao, tiene seis casas y depósitos de planta rectangular o cuadra$\mathrm{da}$, una cocina de verano, compuesta por una pirca de $1 \mathrm{~m}$ de alto sin techo y de planta circular (kancha), un horno de barro, dos corrales, uno para cabras y otro para llamas, una capilla (santuario), tres cuadros de cultivo (rastrojos). La base residencial más pequeña, Vertiente, presenta las siguientes estructuras: seis habitaciones (casas), una cocina de invierno (cocina techada), una cocina de verano sin techar y de planta circular (kancha), un horno de barro (horno para pan) y dos corrales de pirca.

Continuidades históricas. ¿Cuándo se construye una "casa de campo"? Al igual que en muchas otras partes de los Andes (ver p.e., Caro 1985; Custred 1977; Flores Ochoa 1968; Nabeshima 2000; Platt 1986), el casamiento de una pareja es también en Huancar el punto de partida para la fundación de una nueva "casa de campo". "Cuando alguien se casa necesita tener un lugar y su propia hacienda", explica la gente. Según las normas, la nueva "casa de campo" es construida sobre tierras de pastoreo que la famila del esposo -su madre o su padre- han transferido al matrimonio. Sin embargo, los matrimonios no son muy frecuentes. El matrimonio no tiene en el Distrito de Huancar la importancia para la vida social, económica y religiosa de las personas que tiene en otras regiones de los Andes (ver p.e., Allen 1988; Bourque 1995; Brush 1977; Carrasco 1999; Carter 1977; Carter y Mamani 1982; Dransart 2002; Flores Ochoa 1968; Gose 1994; Harris 1986 y 2000a; Isbell 1978 y 1997; Mayer y Bolton 1980; Platt 1986; Rescaniere 2001; Valderrama y Escalante 1998; Wachtel 1990).

14 Como Yacobaccio y colaboradores (1998) no utilizan la terminología local para las estructuras, la hemos agregado en algunos casos entre paréntesis, para facilitar así la comparación entre la situación en Huancar y la situación en Susques.
En los años 1993 y 1999 sólo el 33\% de todas las mujeres con niños menores de 14 años estaban casadas. Vinculado con esta situación el 37\% de las unidades domésticas pastoriles son matrifocales, o sea, tienen un jefe de familia femenino. En estas unidades domésticas no viven hombres adultos (de 14 a 60 años). El gran porcentaje de "madres solteras" no es un desarrollo reciente que corresponde a procesos de desestructuración social. Los datos genealógicos que hemos recopilado y que cubren alrededor de 120 años muestran que son constantes de la organización social en la región.

Aparte del casamiento existen otros dos mecanismos para obtener una "casa de campo". Un mecanismo es "hacerse cargo" de la madre y/o del padre cuando ellos cumplen 60 años. Si una persona se hace cargo de la madre o de los padres cuando ellos envejecieron, recibe como compensación por el cuidado y la responsabilidad a la "casa de campo", a gran parte de los pastoreos y a la mayoría de los puestos y de las aguadas. La norma dice que el hijo o la hija menor deberían hacerse cargo. ${ }^{15}$ Pero, en la práctica, los hermanos prefieren asignar este cargo a una hermana soltera con hijos, porque ella permanece en el lugar y sus hijos pueden colaborar en la economía doméstica. En el lapso 1890-1993 el 45\% de las mujeres solteras con hijos se hizo cargo de su madre y/o su padre.

Otro mecanismo para adquirir una "casa de campo" es la adopción. Si una pareja, no tiene hijos, puede designar a un heredero. El heredero cuidará a la pareja, a la mujer o al hombre que lo adoptó cuando sean viejos. A cambio recibe todos los derechos sobre la "casa de campo", los pastoreos, los puestos y las aguadas. Especialmente aquellas personas entran en arreglos de adopción, porque por diversas razones (p.e., por tener demasiados hermanos o por ser los pastoreos demasiado pequeños) no pudieron recibir pastoreos de sus propias familias. Para el lapso 1890-1993 las genealogías de Huancar muestran cuatro adopciones. Se trata exclusivamente de hombres casados.

15 Esta norma existe también en muchas otras regiones de los Andes; ver p.e., Caro (1985: 68), Carrasco (1999: 98), Custred (1977: 128-129), Flores Ochoa (1968: 232), Platt (1986: 232), Rescaniere (2001: 157). 
Mientras que la construcción de una nueva "casa de campo" requiere de la constitución de un matrimonio, estos dos otros mecanismos - "hacerse cargo" y adopción- implican la transferencia intergeneracional de una "casa de campo" ya existente, sin que fuera necesario para ello contraer matrimonio. Un indicio de la importancia de las transferencias intergeneracionales de "casas de campo" y de las continuidades de uso que implican es la antigüedad de muchas "casas de campo" del Distrito de Huancar. La mayoría de ellas tiene más de 50 años. Según la historia oral el $21 \%$ de las "casas de campo" analizado fue construido entre 1850 (o antes) y 1900, el $45 \%$ entre 1900 y 1950 y el 34\% entre 1950 y 1985.

La descripción bastante detallada de algunas "casas de campo" en un censo ganadero, que se realizó en el año 1937 en el actual Distrito de Huancar, muestra también ciertas continuidades en las estructuras arquitectónicas (Tabla 3).

A pesar de que la base de datos no es grande, el censo de 1937 parece confirmar una tendencia que señalan las personas viejas de Huancar. Ellos cuentan que siempre existieron los elementos que encontramos hoy en día en una "casa de campo": la casa, el corral, la kancha, la cocina, el rastrojo, el santuario, el mojón, etc. Una excepción es la letrina, ya que se trata de un desarrollo reciente que data de principios de los '90. Lo que, sin embargo, fue aumentando en los últimos 50 años es la infraestructura de las "casas de campo". Se incrementó la cantidad de casas y de corrales. La comparación del censo con nuestros propios relevamientos da indicios de este proceso. Así, por ejemplo, el porcentaje de "casas de campo" con cocina se incrementó del $46 \%$ en 1937 al $76 \%$ en 1992/93 (ver Tabla 3) y el porcentaje de "casas de campo" con dos y tres corrales del $15 \%$ al $40.5 \%$ y del $0 \%$ al $4.5 \%$, respectivamente (Tabla 4 ).

También la comparación directa de la infraestructura que posee una determinada "casa de campo"

\begin{tabular}{|lcc|}
\hline Estructura & Casas de campo & $(\%)$ \\
\hline casa(s) & 13 & 100 \\
corral(es) & 13 & 100 \\
kancha & 13 & 100 \\
cocina & 6 & 46 \\
rastrojo & 4 & 31 \\
\hline
\end{tabular}

Tabla 3. Distribución de estructuras en casas de campo de 1937.

\begin{tabular}{|lcr|}
\hline Estructura & Casas de campo & $(\%)$ \\
\hline 2 casas & 5 & 38.5 \\
3 casas & 5 & 38.5 \\
1 casa & 2 & 15 \\
5 casas & 1 & 8 \\
1 corral & 11 & 85 \\
2 corrales & 2 & 15 \\
\hline
\end{tabular}

Tabla 4. Cantidad de casas y corrales de casas de campo en 1937.

en 1937 y la que tiene en 1992/93 sugiere la misma tendencia. Con respecto a la cantidad aumentó el espectro de las casas que puede tener una "casa de campo" (compárense Tabla 2 con Tabla 4). Pero hay que subrayar que el número de las "casas de campo" descritas en 1937 es demasiado bajo como para llegar a conclusiones fehacientes.

Descripciones y fotos históricas de "casas de campo" en la Puna de Atacama como también en la Puna de Jujuy muestran continuidades en el tipo de estructuras que podemos observar hoy en día en las "casas de campo" de Huancar (para las fotos ver Bowman 1924: 301, fig. 113, y Cerri 1903, figs., 42, 44).

"En las paredes de las viviendas hay varios nichos; el principal está ocupado por la imagen de algún santo; en los demás se guardan los objetos más preciados (...) Unos bancos hechos con barro y adosados a la pared sirven de catres, sobre los cuales se disponen cueros de llamas y ovejas. Completan el mobiliario una mesita baja y alguna silla de madera de cardó (...) Como construcciones accesorias pueden mencionarse la cocina, un depósito y los corrales" (Casanova 1936: 269).

"No hay cocina: no la hay en ningún cuarto. Un corralito circular de algo más de un metro de radio, hecho con piedra sin barro ni argamasa, oficia de cocina" (Vicente y Vicente 1942: 126).

"No lejos de la casa, por temor al daño, están el chiquero y los corrales ... Son de pirca, esto es, de paredes de piedra (cantos rodados) sin cemento, altas de un metro y medio y anchas como de sesenta a setenta centímetros ... Los chiqueros son más chicos y suelen tener una parte techada" (Carrizo 1934: 30-31). "Frente al corral está el mojón...” (Carrizo 1934: 31) “... es este un montón 
de piedras en forma de cono, de unos $60 \mathrm{~cm}$ de altura, a manera de apacheta" (Carrizo 1934: 47, cursiva en el original).

Para comparaciones más contundentes de la arquitectura del pastoreo en las tierras altas del Noroeste Argentino harían falta, sin embargo, investigaciones más sistemáticas.

\section{Las "estancias"}

A una distancia de entre 30 minutos a un día de caminata de la "casa de campo" están situados los puestos temporarios, denominados en esta región "estancias". En promedio, cada familia posee cinco "estancias", abarcando el espectro de cero a 11 "estancias". Yacobaccio y colaboradores (1998: 38) mencionan para los alrededores del pueblo de Susques un espectro de dos a siete puestos.
Semejanzas y diferencias entre estancias. El 67\% de las 188 "estancias" que hemos documentado en el Distrito de Huancar consiste básicamente en un corral para ovejas y cabras, el dormidero de las llamas y en un muro circular de piedras o de arbustos como resguardo contra el viento (Figuras 6 y 7). Este círculo, que tiene un fogón (fueguero) en el centro, recibe el nombre de kancha, lo que significa corral. O sea, al igual que los animales también los hombres tienen su corral.

Es importante señalar que tanto la kancha (de los humanos) como el corral (de los animales) pueden ser de piedra o de arbustos (tolas, checales). Esto depende de la disponibilidad local de piedras adecuadas y de arbustos y de la frecuencia de uso de la estancia. Si se trata de una "estancia" muy frecuentada es más común que sea de piedra. El $45.25 \%$ de aquella mayoría de "estan-

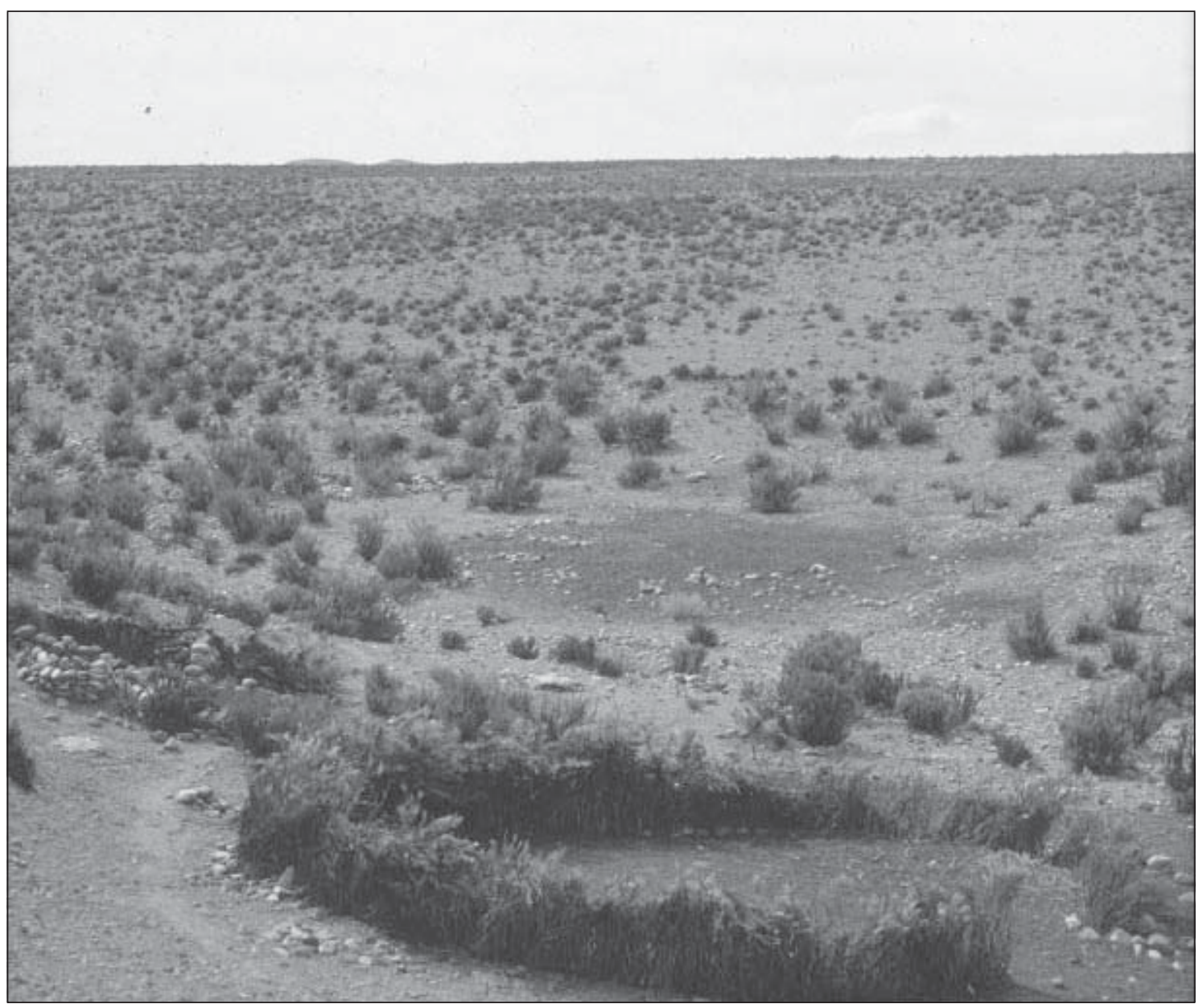

Figura 6. Estancia Filo con corral y kancha de arbusto (tola). 


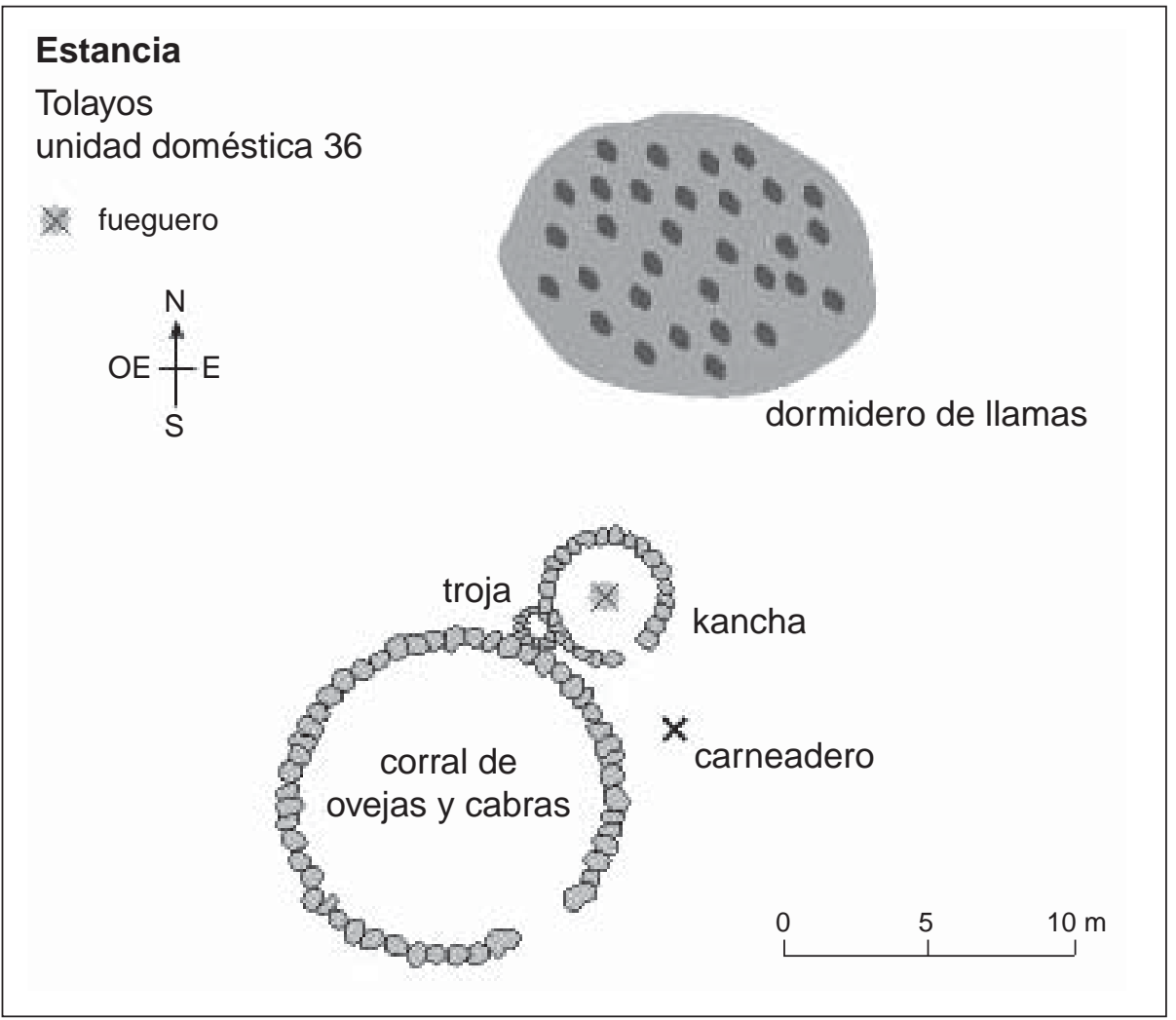

Figura 7. Croquis de la estancia Tolayos (unidad doméstica 36).

cias" del distrito que sólo poseen las estructuras kancha y corral, tienen una kancha y un corral de piedra. Otro $45.25 \%$ de ellas tiene una kancha y un corral de arbustos. El 9.5\% restante o tiene una kancha de piedra y un corral de arbusto o una kancha de arbusto y un corral de piedra.

A diferencia de la kancha de la "casa de campo", la kancha de la "estancia" no solamente es cocina, sino que también es dormitorio. Los pastores duermen allí sobre un cuero de llama o oveja, tapados con una frazada. Muchas veces se encuentra al lado de la kancha una "troja", en la que los pastores depositan durante el día la carne charqueada, para que no la coman perros, zorros o pumas.

El $33 \%$ de todas las 188 "estancias" del distrito tiene, además de la kancha una pequeña choza circular ("rancho") (ver Figuras 8 y 9). El rancho es utilizado como lugar de almacenamiento de vajilla, frazadas y alimentos. Si hay rancho, no hace falta entonces una "troja".
Diez unidades domésticas de Huancar no disponen de ninguna estancia con un rancho. La mayoría de las unidades domésticas restantes sólo tienen una a dos "estancias" con rancho. El relevamiento ha mostrado que para el $87.5 \%$ de las unidades domésticas, la mitad o más de la mitad de sus "estancias" sólo están conformadas por las estructuras básicas corral y kancha.

Yacobaccio y colaboradores (1998: 28), que analizaron 12 sitios temporarios o puestos ("estancias") en los alrededores del pueblo de Susques, describen una situación comparable a la de Huancar. También señalan que sólo pocas "estancias" tienen una casa, aunque no pudieron constatar que esta fuera circular, así como es muy frecuente en Huancar:

"En general, todos los sitios temporarios constan de una sola habitación y un corral adosado, aunque algunos pueden tener hasta dos o tres habitaciones. El 54\% del total de la muestra está construido en pirca seca sin techo ... el $27 \%$ es de pirca, tola y adobe, también sin techo ... y sólo el 


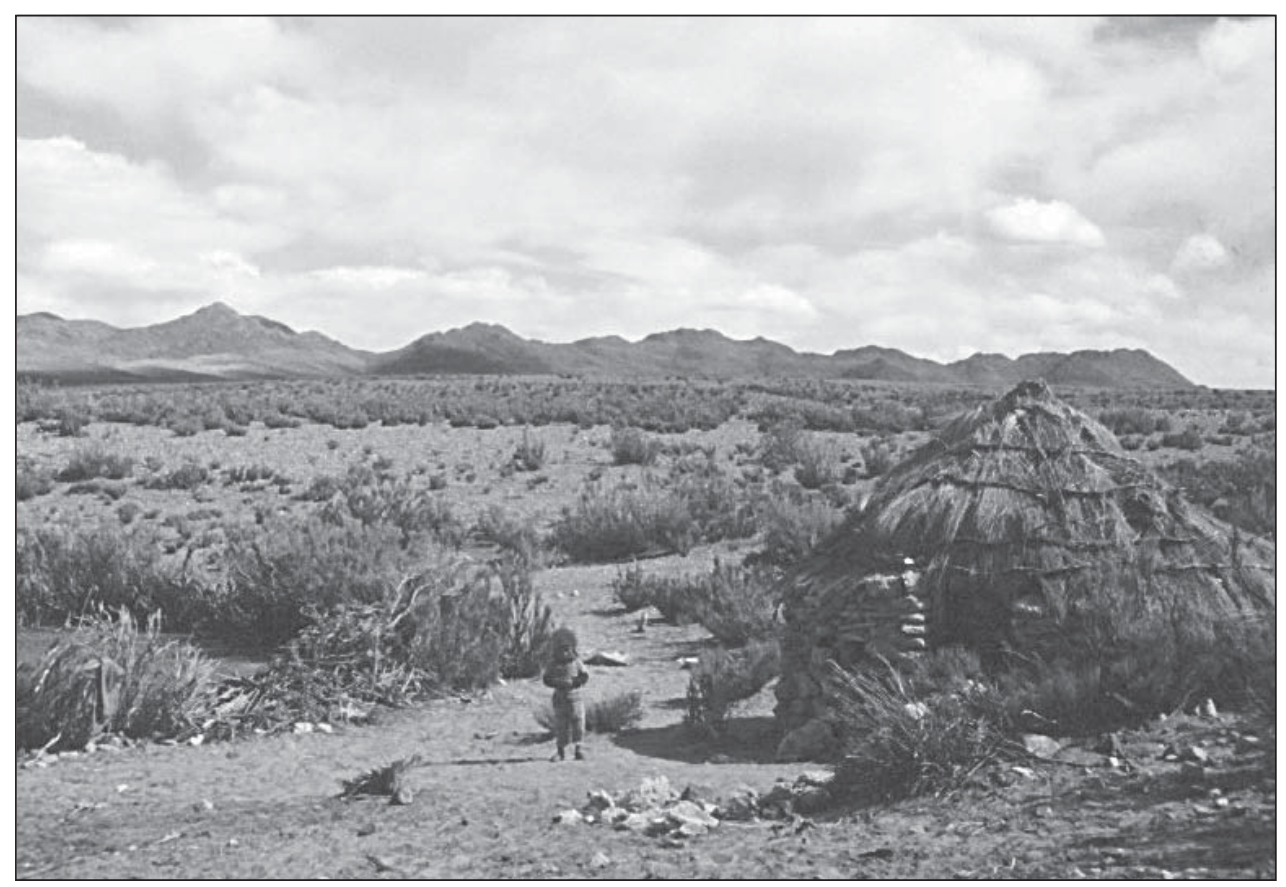

Figura 8. Estancia Panizo con rancho circular.

\section{Estancia}

Piscuno unidad doméstica 14
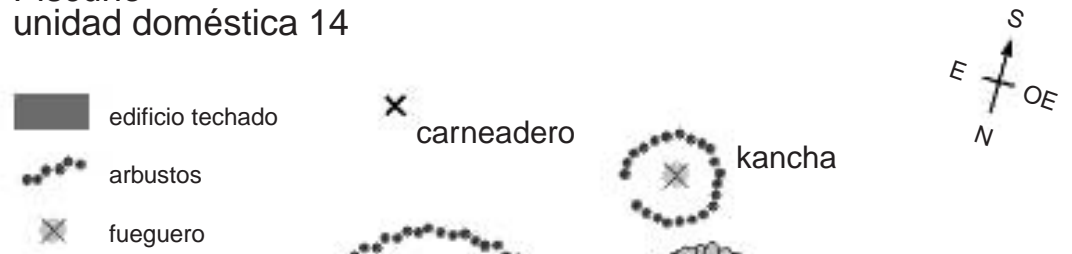

× fueguero
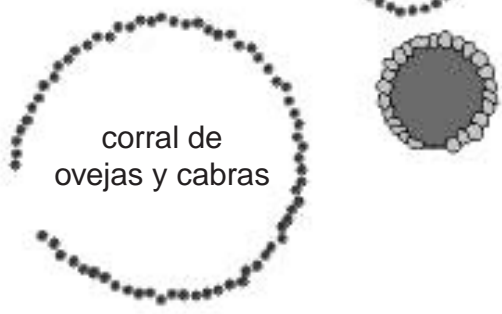
rancho (depósito)

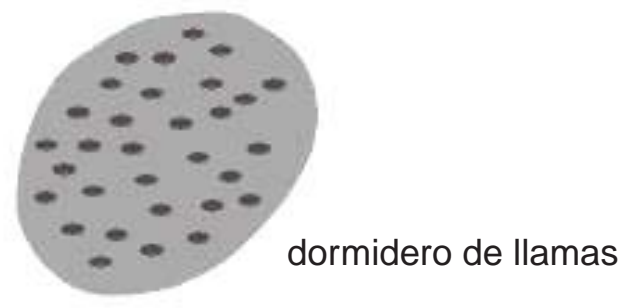

Figura 9. Croquis de la estancia Piscuno. 
$18 \%$ tiene recintos de adobe y techos a dos aguas" (Yacobaccio et al. 1998: 42).

Yacobaccio y colaboradores (1998: 42) determinaron además que "del total (de los sitios temporarios) el 33\% está construido aprovechando cuevas y aleros rocosos". También en Huancar pudimos observar el aprovechamiento de cuevas y aleros como "estancias". Sin embargo, no tenemos números exactos al respecto. A partir de los casos conocidos estimamos que el porcentaje en Huancar es menor al de Susques y giraría alrededor del $15 \%$ de las "estancias".

A nivel comparativo también es interesante mencionar que Yacobaccio y colaboradores (1998: 43) constatan la existencia de "trojas" y "chiqueros" en las "estancias": "En siete de los nueve sitios relevados hay 'chiqueros': pequeñas construcciones de piedra techadas para proteger a los animales recién nacidos y/o para almacenar distintos alimentos".

Hemos visto que en las inmediaciones de una "casa de campo" siempre se encuentra una fuente de agua (vertiente, pozo cavado, etc.). Este no es el caso para las "estancias" del Distrito de Huancar. La mayoría de las veces las personas tienen que recorrer ciertas distancias para acceder al agua. Además, gran parte de las aguadas no son permanentes y se secan durante algunos meses del año, siendo los meses más críticos septiembre, octubre y diciembre.

Continuidades históricas. Un porcentaje importante de las "estancias" tiene 50 o más años de antigüedad. Existe cierta renuencia de las familias de pastores para contruir una "estancia" nueva. Una de las razones que dan es la falta de mano de obra masculina en muchas unidades domésticas. Los hombres son necesarios para la construcción de la kancha, el corral o el "rancho"; trabajo que requiere de fuerzas y habilidades que las mujeres dicen no tener. Otra razón, más fundamental, para la antigüedad de muchas "estancias" es una determinada lógica cultural, que enfatiza las continuidades en el uso del espacio por parte de las familias como un elemento central de las estrategias de manejo de riesgos ambientales.

Existen algunas descripciones históricas de "estancias" en la Puna de Atacama que muestran continuidades en el tipo de construcciones que vemos actualmente en el Distrito de Huancar. ${ }^{16}$ El primer gobernador del Territorio de los Andes, el general italiano Cerri, quien recorrió en 1900 todo el sector oriental de la Puna de Atacama, constata:

"Sus miseras habitaciones son pircas con un techo compuesto de paja y arbustos malamente colocados, con el objeto ostensible de ampararse de los ardientes rayos solares más que de las lluvias raras en esas altas regiones" (Cerri 1903: 41, cursiva en el original).

Y los maestros españoles Vicente, que trabajaron entre 1913 y 1920 en la escuela de Cobres (en las inmediaciones del actual Distrito de Huancar), describen una "estancia" de esta manera:

"En un punto cualquiera de la planicie, había hecho una cavidad rectangular de unos dos metros de largo por uno y medio de ancho y uno de profundidad. Cercóla con una pared de setenta centímetros, la techó con guayas, dejó una abertura para entrar ... Esta era la estancia del mиy bueno y hospitalario Tolaba, espécimen de los habitantes de aquellas alturas" (Vicente y Vicente 1942: 297-298, cursiva en el original).

\section{La "casa en el pueblo"}

En el centro del Distrito de Huancar se encuentra el pequeño pueblo de Huancar (Figura 10). El pueblo fue fundado en 1935 a partir de la construcción de una escuela. Ofrece hoy en día cierta infraestructura estatal: una escuela-albergue, un puesto de primeros auxilios, un puesto de policía (desocupado hace varios años) y la casa de la junta vecinal, en la que se realizan mensualmente las reuniones con todos los pobladores del distrito. También existen en el pueblo una capilla y tres pequeños almacenes.

Todas las familias del distrito poseen una "casa en el pueblo". La infrastructura de las "casas en

16 Para fotos históricas de "estancias" (con y sin "rancho") cerca de Soncor, San Pedro de Atacama y La Poma (borde oriental de la Puna de Atacama, cerca del Valle Calchaquí) véase Bowman (1924: 243, figs. 78, 79, 301, fig. 112). La "estancia" con un "rancho" redondo que Von Rosen (1957: 89) fotografió en 1903 en la Puna de Jujuy es muy parecida a la estancia Panizo del Distrito de Huancar. Sarmiento (1951: 149) escribe sobre la Puna de Jujuy: "El puesto no es más que una choza y un corral en medio del campo". 


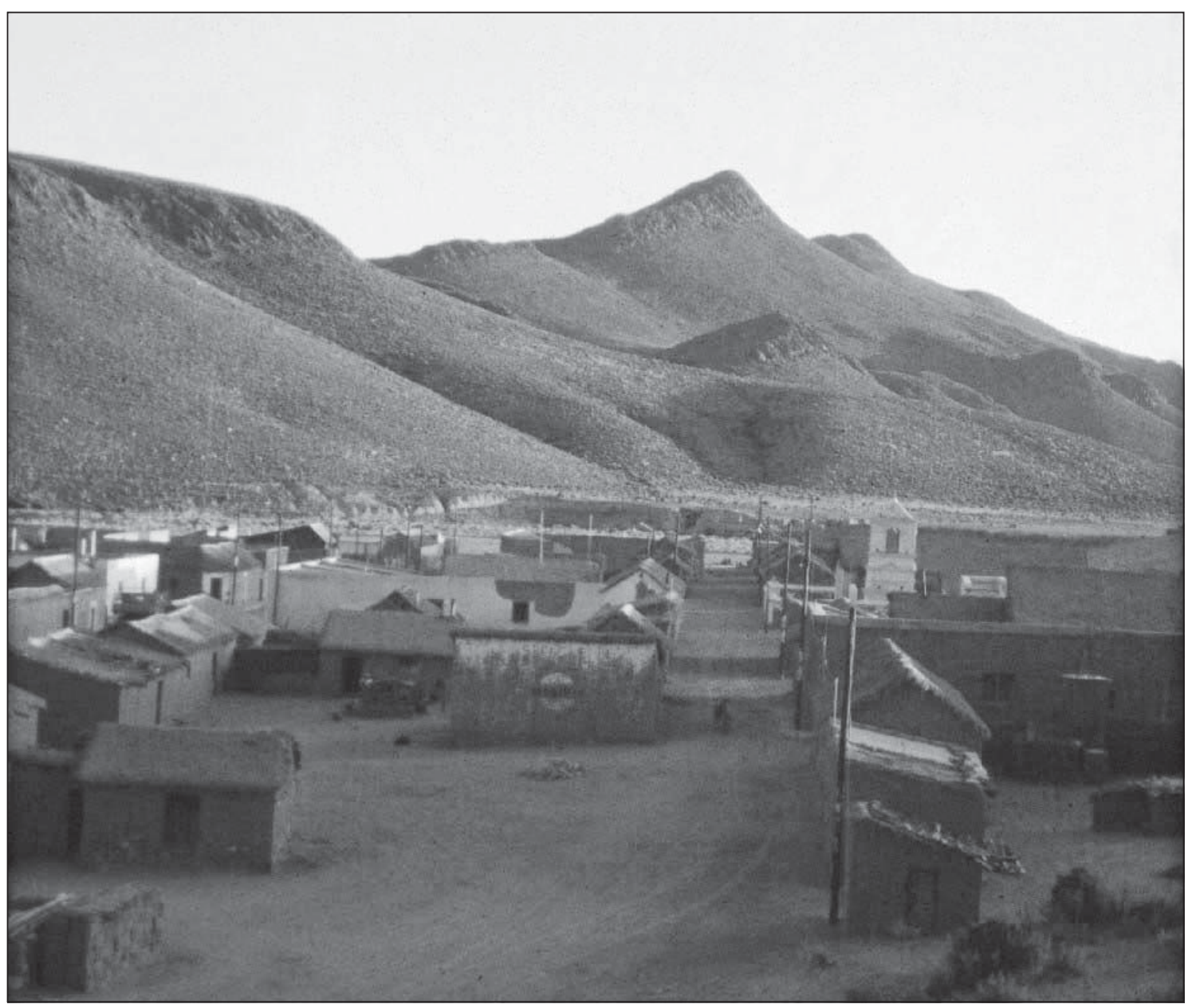

Figura 10. El pueblo de Huancar.

el pueblo" de las familias de pastores es mucho menor que la de aquellas pocas familias que viven permanentemente en el pueblo, porque tienen un empleo público, perciben una jubilación y/o tienen un pequeño almacén. En la mayoría de los casos, las casas de pastores consisten sólo en una o dos "casas de familia", utilizadas como dormitorios y salas de estar, y una kancha con un fueguero para poder cocinar.

La influencia de la arquitectura urbana se nota más en las "casas en el pueblo" que en las "casas de campo". Las casas nuevas poseen techos de tinglado y puertas y ventanas de carpintería. Siguen teniendo paredes de adobe, pero muchas de ellas ya no tienen base de piedra. Las paredes de las casas viejas del pueblo de Huancar o tienen base de piedra y adobe en la parte superior, o están hechas -al igual que las "casas de campo"- totalmente de piedra. Tienen una apariencia similar a las casas que describe Boman (1908: 429) a principios del siglo XX en el pueblo de Susques ${ }^{17}$ :

"Les maisons sont rectangulaires, toutes à peu près de mêmes dimensions, environ $6 m$ de longueur sur $3 \mathrm{~m}$ de largeur, bàties en adobes (briques crues séchées au soleil). La toiture en chaume (paja brava) est supportée par un comble à deux versants. Le faîtage et les chevrons reposent directament sur les pignons et sur les murs. Faîtage, arbalétriers,

\footnotetext{
${ }^{17}$ Para otras descripciones históricas de casas en la Puna de Atacama véanse Cerri (1903: 58), Von Rosen (1957: 7778) y Vicente y Vicente (1942:9 8-100, 126-127); para la Puna de Jujuy véanse Carrizo (1934: 29-30) y Casanova (1936: 296). Las descripciones transmiten una buena impresión del interior de muchas de las casas viejas en el pueblo y de muchas "casas de campo".
} 
chevrons et pannes sont tous en bois de cactuscierge (Cereus), seul bois de construction qui existe à Susques. L'extrémité supérieure des chevrons est fixée au faîtage au moyen d'encoches reenforcées par des attaches en peau. Tout les autres pièces sont simplement assemblées au moyen de lanières en peau. (...) Il n'y a pas d'ouverture dans le toit pour la fumée, parce qu'on ne fait qu' accidentellement du feu dans la maison. Le foyer, une simple plate-forme circulaire en terre, bordée de pierres, a sa place dans un petit hangar en dehors de la maison, lequel est également pourvu d' un toit en chaume et fermé, au moins de trois côtes, par des murs" (cursiva en el original).

Las familias de pastores de Huancar visitan sólo esporádicamente a su "casa en el pueblo". Ocasiones para ir al pueblo de Huancar son, por ejemplo, la compra de mercadería, las reuniones de la Junta Vecinal o de la Cooperadora Escolar y las fiestas patronales. A diferencia de la "casa de campo" nunca se reúne la familia completa en ella, sino que únicamente va un miembro de la familia o van unos pocos miembros de la familia. Esto no significa que la "casa en el pueblo" sea percibida como una estructura aislada o hasta opuesta a la "casa de campo". Más bien forma parte del sistema de movilidad espacial de las unidades domésticas pastoriles. Al igual que los puestos ("estancias") también la "casa en el pueblo" no es habitada permanentemente, sino que sólo temporariamente según fines muy específicos (p.e., una reunión de la Junta Vecinal). También las descripciones históricas constatan esta función de la "casa en el pueblo". Los viajeros naturalistas, los exploradores militares y los administradores estatales que recorrieron el sector oriental de la Puna de Atacama señalan que los pueblos están abandonados y las casas vacías, porque los indígenas se encuentran en los cerros, cuidando a sus rebaños (Bertrand 1885; Boman 1908; Bowman 1924; Cerri 1903; Holmberg 1900; Von Rosen 1957; Vicente y Vicente 1942).

\section{El uso económico y simbólico del espacio}

La movilidad espacial es un instrumento efectivo de adaptación a fluctuaciones imprevisibles en las condiciones climáticas y ecológicas. En comparación con sectores más húmedos de las tierras altoandinas, la movilidad espacial con los rebaños es bastante alta en Huancar (ver Flores Ochoa 1968; Orlove 1981; Palacios Ríos 1987; West
1983). Aproximadamente cada tres semanas las familias se trasladan con sus llamas, ovejas y cabras de un puesto a otro o regresan a la "casa de campo". Los pastores mencionan una serie de razones que los llevan a cambiar de lugar: un factor es el agotamiento de las pasturas, ya sea una merma general de las pasturas o de algunas plantas consideradas como muy nutritivas; otros factores son la lejanía de fuentes de agua o su creciente desecación, el descenso brusco de la temperatura, la irrupción de heladas, nevadas o fuertes lluvias, la aparición de fuertes vientos de polvo o simplemente el aburrimiento de la "hacienda".

Cada unidad doméstica tiene su propio ciclo de rotación, determinado por las características de sus tierras de pastoreo y las correspondientes fuentes de agua, por el tamaño y la composición de sus rebaños y por la mano de obra disponible. Los ciclos tienen dos características en común: primero, los movimientos abarcan un espacio relativamente restringido (como máximo las familias caminan con el ganado dos días para llegar al nuevo lugar de pastoreo). Y, segundo, se aprovechan diferentes ecozonas distribuidas por distintos niveles altitudinales. Sin embargo, no existe en los movimientos verticales un patrón homogéneo para todas las unidades domésticas del Distrito de Huancar. Las familias que tienen sus "casas de campo" en la serranía occidental (al oeste del río Pastos Chicos) bajan durante el invierno (mayo a octubre) a las pequeñas quebradas transversales (doblado), mientras que las unidades domésticas que tienen sus "casas de campo" en la serranía oriental (al este del río Pastos Chicos) suben en esos meses a sus "estancias" más altas. En cambio, en el verano (noviembre a abril) las familias de la serranía occidental suben a sus estancias más altas, mientras que las familias de las serranías orientales bajan en estos meses a las estancias que tienen cerca del río Pastos Chicos. La unidad doméstica 10, por ejemplo, tiene su "casa de campo" en Barrancas, en la serranía oriental. La gente de Barrancas se desplaza en el verano con su ganado entre las "estancias" 6, 7, 8 y la "casa de campo" en Barrancas. En el invierno suben con el ganado a las "estancias" 1, 2, 3, 4 y 5 (Figura 11).

Hay que destacar que siempre se desplaza todo el grupo familiar con el ganado. Por lo tanto, no se puede hablar en el caso de Huancar de un sistema transhumante, sino más bien de un sistema seminómade. Debido a este alto grado de movi- 


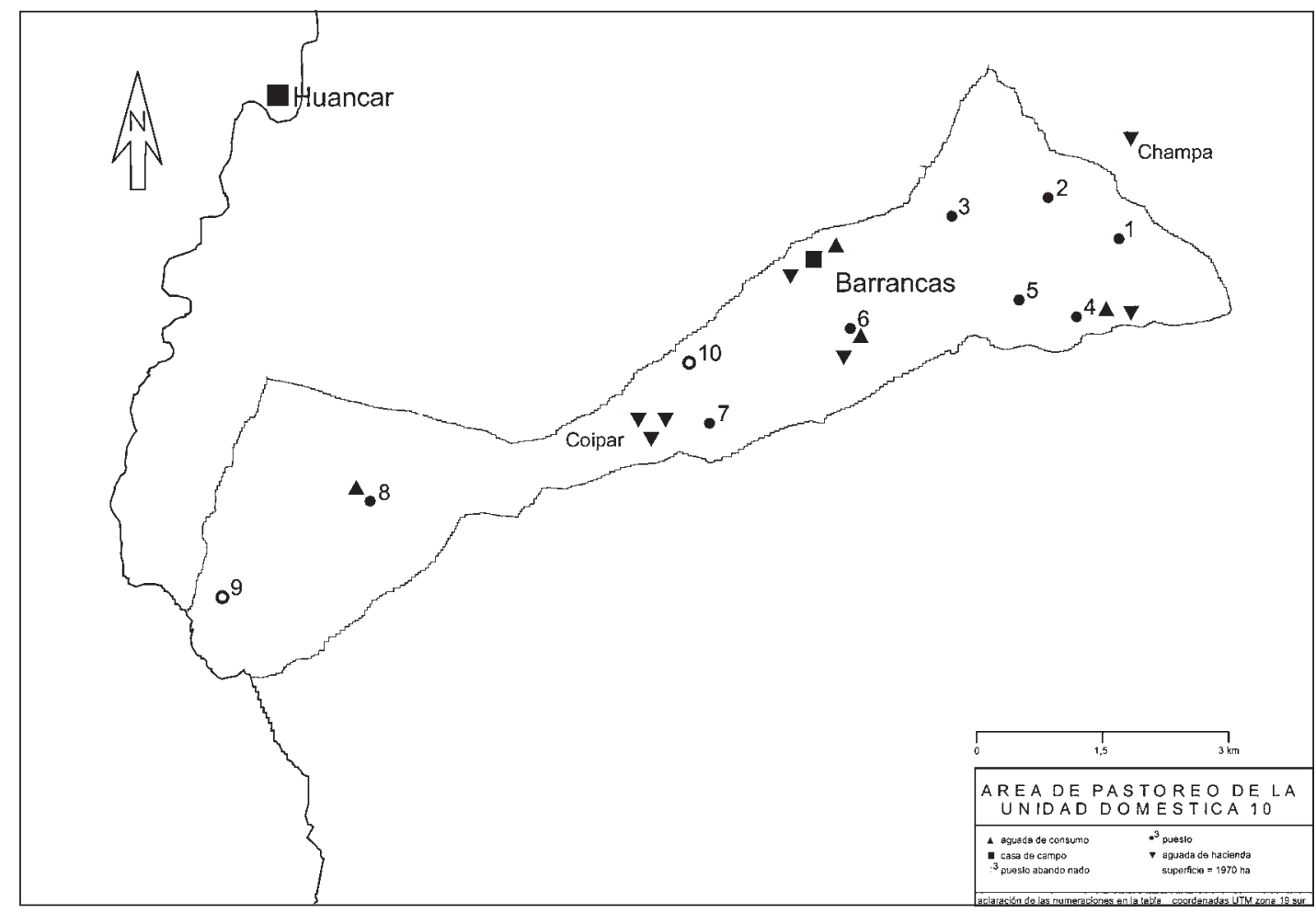

Figura 11. Área de pastoreo de la unidad doméstica 10: Casa de campo Barrancas, estancias y aguadas para personas y animales.

lidad, una familia no vive más que dos o tres meses al año en su "casa de campo". A pesar de esta situación la "casa de campo" exhibe una infrastructura considerable, mucho mayor que la de los puestos ("estancias") o de la pequeña casa, que cada familia de pastores tiene en el pueblo de Huancar.

La "casa de campo" es el lugar en el cual se realizan actividades excepcionales con el ganado, como la esquila y la castración. Es además el lugar en el que se llevan a cabo las fiestas sociales de la familia y sus rituales más importantes (señalada $1^{\circ}$ de agosto: el día de la Pachamama, el día de los respectivos santos de los animales). Debido a la densidad de prácticas culturales, la "casa de campo" es un lugar impregnado de historia. Los edificios y estructuras materiales son percibidos como almacenes materiales de prácticas culturales pasadas. Ellos pueden "contar historias". Por lo tanto, la "casa de campo" es un importante símbolo de la identidad de una unidad doméstica. Así, por ejemplo, el par de mazorcas atado debajo de una viga del dormitorio o de la "ramada" evoca caravanas de intercambio de años anteriores. Otro ejemplo para la condensación de información en objetos o estructuras es el "mojón". Cada año, durante el ritual de la señalada, se agregan piedras al montículo. De esta manera el "mojón" documenta de una forma socialmente visible el crecimiento de los rebaños de una familia. Es un indicador importante del éxito económico de una familia de pastores.

Los esquemas de relacionamiento con el medio ambiente y los significados del espacio familiar son reproducidos y reforzados durante actos rituales. Estos abarcan desde las ch'allas cotidianas que acompañan muchas actividades económicas hasta rituales complejos como la señalada. Durante cada ofrenda que se hace a la pachamama es recreado todo el microcosmos del uso pastoril del espacio por parte de una unidad doméstica. La persona que realiza la ofrenda menciona la "casa de campo" y de este a oeste los lugares de las "estancias" y de las aguadas de la familia. De esta manera el control práctico que tienen los miembros de una unidad doméstica sobre un determinado territorio es estabilizado a través del control simbólico del territorio. 
Los marcadores materiales de las clasificaciones del territorio familiar - los edificos de la "casa de campo", las "estancias", los corrales, las aguadas, los "mojones", los "despachos, etc.- tejen una densa malla de las relaciones entre los hombres y su medio ambiente. Los miembros de una familia han acumulado a lo largo del tiempo amplios conocimientos sobre el medio ambiente, las relaciones con los principales componentes del espacio y sobre la dinámica del entorno natural, en el que experimentan su vida cotidiana. Estos conocimientos tienen un alto grado de exclusividad. Así, por ejemplo, los conocimientos de muchos topónimos no trascienden el ámbito familiar o son sólo parcialmente compartidos por unidades domésticas vecinas. Como la gente de Huancar enfatiza, sólo es importante conocer los nombres de aquellos lugares que tienen un impacto, un efecto concreto sobre los miembros humanos y no-humanos de la unidad doméstica. Sólo se conoce a aquellos componentes del medio ambiente que tienen relevancia para la toma de decisiones. No existe un interés general en memorizar nombres de lugares, con los que no se tiene ninguna relación práctica, ningún vínculo performativo. A través de sus interacciones directas con su entorno natural los individuos adquieren, reproducen y refuerzan sus conocimientos ambientales.

Esta es la razón por la cual muy pocos habitantes pueden nombrar los límites de todo el Distrito de Huancar. Lo que sí conocen son los límites exactos de sus pastoreos y de los pastoreos colindantes, ya que esta información es de importancia para el uso de recursos y la prevención de conflictos con vecinos. Cuando las personas comparan o delimitan unidades domésticas hablan de las tierras de pastoreo, de los puestos, aguadas y rebaños de una unidad doméstica como de un conjunto. En estos contextos de orientación exterior, que trascienden el ámbito netamente familiar, el espacio familiar constituye una unidad homogénea. En cambio, dentro del marco de una unidad doméstica esta unidad se fragmenta en espacios con significados divergentes (ver también Göbel, en prensa a). Determinadas partes del entorno y de la infraestructura material (p.e., el corral) adquieren, entonces, una impronta de género. La impronta de género de ciertos sectores del espacio familiar está vinculada a la marcada división de trabajo por género, que existe en la economía local.

\section{Impronta de género del espacio}

La división de trabajo por género en Huancar se caracteriza por el contraste entre la fijación local de las mujeres y la orientación exterior y amplia movilidad espacial y social de los hombres. La principal competencia económica de las mujeres es el manejo general de las llamas, ovejas y cabras y su control diario. A pesar de que las niñas y mujeres son involucradas desde temprana edad en el cuidado de los rebaños, existe en cada unidad doméstica una pastora principal que es reconocida socialmente como la mujer encargada de la "hacienda" de esa unidad doméstica. Se trata de la esposa del jefe del hogar o de la mujer a cargo del hogar, en caso de que no viviera ningún hombre adulto en él. La pastora principal no solamente toma las decisiones finales sobre las actividades cotidianas y extraordinarias con los animales, sino que también dirige los rituales relacionados con el rebaño (p.e., la señalada, el festejo de los santos de los animales, las ch'allas del $1^{\circ}$ de agosto).

Las áreas de trabajo más prominentes de los hombres son la articulación de la producción pastoril con el "mundo de afuera" y la suplementación de la producción pastoril con otras formas de ingreso. Por un lado, los hombres organizan caravanas de intercambio con burros y mulas para adquirir productos agrícolas (para más información ver Göbel 1998a y 1998b). El cultivo de plantas es casi imposible en este sector tan árido de la puna. Ya hemos mencionado que sólo algunas pocas unidades domésticas tienen unos pequeños "rastrojos", que son trabajados por los hombres. Esto hace que el intercambio económico sea de importancia elemental en la economía pastoril de Huancar. En general, los componentes básicos de la dieta cotidiana como maíz, papas y habas, tienen que ser traídos desde lugares más bajos como la quebrada de Humahuaca, los valles Calchaquíes o el oasis chileno de Toconao cerca de San Pedro de Atacama. Los productos agrícolas son comprados o trocados por productos de la puna como tejidos a telar y de aguja, quesos, carne, cueros, sal, hierbas medicinales, coipa y bloques de sal de las Salinas Grandes.

El otro eje de la movilidad masculina es el trabajo temporario en las minas y en las plantaciones de tabaco y fruta de las tierras bajas. Como los hombres combinan a lo largo de un año diversos 
contratos de trabajo con múltiples viajes de intercambio, no se encuentran durante muchos meses en sus hogares.

Todas las actividades, los objetos materiales y los conocimientos relacionados con la realización de viajes de intercambio y el trabajo temporario en minas y plantaciones son de dominio masculino. Así, por ejemplo, los burros y las mulas constituyen importantes símbolos de estatus masculino. También el trabajo en el telar, que está estrechamente vinculado a las caravanas de intercambio, es considerado un trabajo de hombres. De la misma manera el conocimiento de las sendas que llevan a minas o a destinos de viajes de intercambio y el conocimiento de los tramos que se encuentran fuera del distrito son de dominio masculino.

Debido a su orientación exterior los hombres poseen conocimientos espaciales más amplios y más diversos que las mujeres. Los viajes de intercambio y el trabajo temporario en minas y plantaciones no solamente les han permitido conocer una gama mucho más amplia de ecozonas, sino también de experimentar contextos sociales más diversos. Las mujeres, en cambio, tienen conocimientos más detallados sobre los pastoreos, particularmente sobre el área de pastoreo de la familia y su dinámica a lo largo del año. Vemos entonces que la marcada división de trabajo en la economía determina notables diferencias en los conocimientos espaciales (Göbel, en prensa a).

Pero, ¿qué impacto tienen las diferencias de género sobre el espacio adscrito a una unidad doméstica? Los edificios o las instalaciones centrales de una "casa de campo" -las "casas de familia", la "ramada", la cocina techada, la kancha, el santuario- no son vistos como lugares que tengan una impronta específica de género. Esto no es el caso para el "rastrojo" o para el telar. Ambos son percibidos como espacios claramente masculinos. Tanto el "rastrojo" como el telar son adscritos a los hombres que trabajan allí. Siempre se dice: "Este es el 'rastrojo' de don ..." o "esto es el telar de don ...".

En situaciones cotidianas los corrales son definidos como los sitios en los que pernoctan ovejas y cabras; como sus dormitorios. En consecuencia siempre se los relaciona con una mujer; al ser de la pastora principal, uno diría: "Este es el corral de doña ...". Esta adscripción puede cambiar en contextos rituales. Allí el corral ya no es más un espacio femenino, sino que pasa a conformar el espacio "sagrado" de la unidad doméstica. Mientras que, por ejemplo, en contextos cotidianos está absolutamente prohibido matar un animal dentro del corral, durante la señalada se sacrifica un animal en el centro del mismo.

En correspondencia con los estrechos vínculos que la gente de Huancar establece entre las mujeres y la producción pastoril, los puestos, las aguadas y los pastoreos constituyen espacios femeninos. Siempre se habla de ellos en términos generales como los puestos, los pastoreos o las aguadas de "doña ....", o sea de la pastora principal. Tanto las estructuras materiales como el espacio, en el que se desenvuelven actividades pastoriles, son percibidos como el espacio doméstico de las mujeres; el área que ellas controlan. Por lo tanto, el área doméstica no se restringe en Huancar a la casa o sus inmediaciones (ver también Göbel, en prensa a).

\section{Comentarios finales}

El pastoreo de Huancar está vinculado a un sistema complejo de asentamientos. Este sistema está conformado por la "casa de campo" -y todo el conjunto de estructuras que aglomera-, por las numerosas "estancias" que cada familia posee y por su "casa en el pueblo". El relevamiento de este sistema de asentamientos es de importancia fundamental para poder comprender los esquemas de uso y significados del espacio. Hemos visto que en Huancar el eje central de la organización social y económica del espacio no es la comunidad, sino que la unidad doméstica. La centralidad de esta para los conocimientos ambientales pone en duda la primacía del "prisma comunitario" a través del cual muchos estudios andinos analizan el medio ambiente. Pudimos determinar patrones comunes entre las unidades domésticas de Huancar (p.e., elementos básicos que componen el sistema de asentamientos, normas compartidas para el uso del espacio), pero también detectamos un notable grado de variabilidad entre ellas (p.e., en los patrones de movilidad). La diversidad y flexibilidad es otra característica de la organización social y económica del espacio en este sector tan árido de las tierras altoandinas.

A modo de conclusión queremos destacar tres aspectos que el presente trabajo sobre la arquitec- 
tura del pastoreo en Huancar ha puesto de relieve y que requieren de un análisis más amplio: en primer lugar el trabajo señala la relevancia que en Huancar tienen las prácticas económicas para los significados del espacio. En segundo lugar, pone de manifiesto que, en las interacciones de los habitantes de Huancar con el medio ambiente, los aspectos económicos, sociales y simbólicos están estrechamente entrelazados. Vemos entonces que no se puede desvincular el uso económico del espacio de su control social y simbólico. En tercer lugar, los datos muestran la multiplicidad y relatividad de los significados del espacio, puesto que según el respectivo contexto interactivo el espacio posee para los agentes diferentes significados.

Agradecimientos Los diversos trabajos de campo en Huancar (años 1991-1993, 1994, 1995, 1996-1997, 1999, 2000) fueron financiados por la Deutsche Forschungsgemeinschaft (DFG), Bonn, el Deutsche Akademische Austauschdienst (DAAD), Bonn; y la Fondation Fyssen, Paris. Mi más profundo reconocimiento va hacia los pobladores de Huancar, por su amistad, su voluntad de dejarme participar en sus vidas y su incansable paciencia con mis preguntas.

\section{REFERENCIAS CITADAS}

ALLEN, C. J., 1988. The hold life has. Coca and cultural identity in an Andean community. Smithsonian Institution Press, Washington D. C.

ASTVALDSSON, A., 1997. Las voces de los Wak'a. Fuentes principales del poder político aymara. CIPCA, La Paz.

BARFIELD, T. J., 1993. The nomadic alternative. PrenticeHall, Englewood Cliffs.

BASTIEN, J. W., 1978. Mountain of the condor. Metaphor and ritual in an Andean ayllu. West Publishing, Nueva York.

BERTRAND, A., 1885. Memoria sobre las cordilleras del desierto de Atacama y regiones limítrofes. Imprenta $\mathrm{Na}-$ cional, Santiago.

BIESELE, M., R. HITCHCOCK y P. SCHWEITZER (Eds.), 1999. Hunter-gatherers in the modern world: Conflict, resistance, and self-determination. Berghahn, Providence.

BOLIN, I., 1998. Rituals of respect. The secret of survival in the High Peruvian Andes. University of Texas Press, Austin.

BOMAN, E., 1908. Antiquités de la Région Andine de la Republique Argentine et du désert d'Atacama. Imprimerie Nationale, Paris.

BONAVIA, D., 1996. Los camélidos sudamericanos. Una introducción a su estudio. IFEA, Lima.

BOURQUE, N., 1995. Savages and angels: The spiritual, social and physical development of individuals and households in Andean life-cycle festivals. Ethnos 60: 99-114.

BOWMAN, I., 1924. Desert trails of Atacama. American Geographical Society, Nueva York.
BROWMAN, D., 1974. Pastoral nomadism in the Andes. Current Anthropology 15: 188-196.

-1989. High altitude camelid pastoralism of the Andes. En The world of pastoralism: Herding systems in comparative perspective, J. Galaty y D.L. Johnson (Eds.), pp. 323-352. Guilford, Londres.

BRUSH, S. y D. GUILLET, 1985. Small-scale agro-pastoral production in the Central Andes. Mountain Research and Development 5: 19-30.

BRUSH, S., 1977. Kinship and land use in a Northern Sierra community. En Andean kinship and marriage, R. Bolton y E. Mayer (Eds.), pp. 136-152. AAA, Washington.

CARDENAS, D. H., 1990. Estudio del sistema pastoril de camélidos en Condoroma: Tres casos de manejo de un distrito. En Agricultura andina: Unidad y sistema de producción, M. Eresue, J. M. Gastellu et al. (Eds.), pp. 261-278, Lima.

CARO, D., 1985. "Those who divide us". Resistance and change among pastoral ayllus in Ulla Ulla, Bolivia. UMI, Ann Arbor.

CARRASCO, A. M., 1999. Constitución de género y ciclo vital entre los aymara contemporáneos del norte de Chile. Chungará 30: 87-103.

CARRIZO, J. A., 1934. Cancionero popular de Jujuy. Universidad Nacional de Tucumán, Tucumán.

CARTER, W., 1977. Trial marriage in the Andes? En Andean kinship and marriage, R. Bolton y E. Mayer (Eds.), pp. 177-216. AAA, Washington.

CARTER, W. E. y M. MAMANI, 1982. Irpa Chico. Individuo y comunidad en la cultura aymara. Juventud, La Paz. 
CASANOVA, E., 1936. El altiplano andino. En Historia de la Nación Argentina. Vol. 1: Tiempos prehistóricos y protohistóricos, R. Levene (Ed.), pp. 251-275. Librería y Editorial El Ateneo, Buenos Aires.

CERRI, D., 1903. El territorio de los Andes (República Argentina). Reseña geográfica descriptiva. Taller Tipográfico de la Penitenciaría Nacional. Buenos Aires. (Edición facsimilar 1992, UNJU, Jujuy).

CLUTTON-BROCK, J. (Ed.), 1989. The walking larder: Patterns of domestication, pastoralism, and predation. Hyman, Londres.

CRIBB, R., 1991. Nomads in archaeology. Cambridge University Press, Cambridge.

CUSTRED, G., 1977. Peasant kinship, subsistence and economics in a high altitude Andean environment. En Andean kinship and marriage, R. Bolton y E. Mayer (Eds.), pp. 117-135. AAA, Washington.

DRANSART, P., 2002. Earth, water, fleece and fabric. An ethnography and archaeology of Andean camelid herding. Routledge, Londres.

DYSON-HUDSON R. y N. DYSON-HUDSON, 1980. Nomadic pastoralism. Annual Review of Anthropology 9: 15-61.

FLANNERY, K., J. MARCUS y R. G. REYNOLDS, 1989. The flocks of the Wamani. A study of llama herders on the Punas of Ayacucho, Peru. Academic Press, Nueva York.

FLORES OCHOA, J. A., 1968. Los pastores de Paratía. Instituto Indigenista Interamericano, Lima.

-1977. Pastores de puna: Uywamichiq punarunakuna. Instituto de Estudios Peruanos, Lima.

-1983. Pastoreo de llamas y alpacas en los Andes - balance bibliográfico. Revista Andina 1: 175-218.

_-1988. Llamichos y paqocheros: Pastores de llamas y alpacas. Editorial Universitaria, Cuzco.

FLORES OCHOA, J. A. y Y. KOBAYASHI (Eds.), 2000. Pastoreo altoandino. Realidad, sacralidad y posibilidades. Plural, La Paz.

FRATKIN, E., 1997. Pastoralism: Governance and development issues. Annual Review of Anthropology 26: $235-261$.

FRATKIN, E., K. A. GALVIN y E. A. ROTH (Eds.), 1994. African pastoral systems. An integrated approach. Lynne Rienner Publisher, Boulder.

GALATY, J. y D. L. JOHNSON (Eds.), 1990. The world of pastoralism. Herding systems in comparative perspective. Guilford and Belhaven Press, Londres.

GÖBEL, B., 1997. "You have to exploit luck": Pastoral household economy and the cultural handling of risk and uncertainty in the Andean highlands. En Risk and uncertainty in pastoral societies, B. Göbel y M. Bollig (Eds.), pp. 37-53. (Special Issue. Nomadic Peoples, NS, 1). Berghahn, Oxford.

1998a. Risk, uncertainty and economic exchange in a pastoral community of the Andean highlands (NWArgentine). En Kinship, networks and exchange, T. Schweizer y D. White (Eds.), pp. 158-77. Cambridge University Press, Cambridge.

_1998b. "Salir de viaje": Producción pastoril e intercambio económico en el Noroeste Argentino. En 50 años de Estudios Americanistas en la Universidad de Bonn. Nuevas contribuciones a la arqueología, etnohistoria, etnolingüística y etnografía de las Américas. 50 Years Americanist Studies at the University of Bonn. New contributions to the archaeology, ethnohistory, ethnolinguistics and ethnography of the Americas, S. DedenbachSalazar, C. Arellano, E. König y H. Prümers (Eds.), pp. 867-891. (Bonner Amerikanistische Studien 30). Verlag Anton Saurwein, Markt Schwaben.

-2001a. The symbolism of llama breeding in NorthWestern Argentina. En Progress in South American camelids research. Proceedings of the 3rd European Symposium and Supreme European Seminar. Universität Göttingen, Göttingen (1999), M. Gerken y C. Renieri (Eds.), pp. 175-180. (EAAP publication 105). Wageningen Pers.

_-2001b. El ciclo anual de la producción pastoril en Huancar (Jujuy, Argentina). En El uso de los camélidos a través del tiempo, G. Mengoni, D. Olivera y H. Yacobaccio (Eds.), pp. 91-115. GZC-ICAZ-Ediciones del Tridente, Buenos Aires.

_-En prensa a. Identidades sociales y medio ambiente: La multiplicidad de los significados del espacio en la Puna de Atacama. Cuadernos del Instituto Nacional de Antropología 19.

_-En prensa b. Identidad y periferia: Las caras de la identidad en Huancar (Puna de Atacama). Mosaico. Revista de Ciencias Sociais.

-En prensa c. Dangers, experience, and luck: Handling uncertainty in the Andes. En Culture and the changing environment. Uncertainty, cognition, and risk management in cross-cultural perspective, M. Casimir y U. Stahl (Eds.). Berghahn, Oxford.

GOSE, P., 1994. Deathly waters and hungry mountains. Agrarian ritual and class formation in an Andean town. University of Toronto Press, Toronto.

GRESLOU, F., 1989. Visión y crianzas campesinas de los animales andinos. En Crianza de llamas y alpacas en los Andes. Proyecto Alpacas - Convenio INIAA - CORPUNO. COTESU, pp. 11-44. PRATEC - Proyecto Andino, Lima.

GUNDERMANN, H., 1984. Ganadería aymara, ecología y forrajes: Evaluación regional de una actividad productiva andina. Chungará 12: 99-124. 
HARRIS, O., 1986. From asymmetry to triangle: Symbolic transformations in northern Potosí. En Anthropological History of Andean polities, J. Murra, N. Wachtel, y J. Revel (Eds.), pp. 260-279. Cambridge University Press, Cambridge.

2000. To make the earth bear fruit. Ethnographic essays on fertility, work and gender in highland Bolivia. Institute of Latin American Studies, Londres.

HOLMBERG, E., 1900. Viaje a la Gobernación de los Andes (Puna de Atacama). Imprenta de La Nación, Buenos Aires. (Edición facsimilar: 1988, UNJU, Jujuy).

INAMURA, T., 1981. Adaptación ambiental de los pastores altoandinos en el sur del Perú. Simbiosis económico-social con los agricultores. En Estudios etnográficos del Perú Meridional, S. Masuda (Ed.), pp. 65-85. Universidad de Tokio, Tokio.

INGOLD, T., D. RICHES y J. WOODBURN (Eds.) 1988. Hunters and gatherers, Vol. I, History, evolution and social change. Berg, Oxford.

ISBELL, B. J., 1978. To defend ourselves: Ecology and ritual in an Andean village. University of Texas Press, Austin.

-1997. De inmaduro a duro: Lo simbólico femenino y los esquemas andinos de género. En Más allá del silencio. Las fronteras de género en los Andes, D. Arnold (Ed.), pp. 253-301. CIASE - ILCA, La Paz.

KELLY, R. 1995. The foraging spectrum. Diversity in huntergatherer lifeways. Smithsonian Institution Press, Washington D. C.

KHAZANOV, A. M., 1994. Nomads and the outside world. University of Wisconsin Press, Wisconsin, Madison. ( $2^{\mathrm{a}}$ edición).

LEE, R. B. y R. DALY (Eds.), 1999. The Cambridge Encyclopedia of hunters and gatherers. Cambridge University Press, Cambridge.

LEE, R. B. y I. DE VORE, 1968. Man the hunter. Aldine, Chicago.

LOPEZ ARANDA, P., 1937. El Territorio Nacional de los Andes. Boletín del Ministerio Nacional de Educación. Buenos Aires.

MARTINEZ, G., 1975. Características de orden antropológico y socioeconómico de la comunidad de Isluga (I Región). Norte Grande 1 (3-4): 403-426.

-1976. El sistema de los uywiris en Isluga. Anales a la Universidad del Norte 10: 255-327.

MAYER, E. y R. BOLTON (Eds.), 1980. Parentesco y matrimonio en los Andes. PUCP, Lima.

MC CORKLE, C., 1987. Punas, pastures, and fields: Grazing strategies and the agropastoral dialectic in an indigenous Andean community. En Arid land use strategies and risk management in the Andes: A regional anthropological perspective, D. Browman (Ed.), pp. 57-80. Westview, Boulder.

NABESHIMA, S., 2000. "También queremos cultivar papales": Un caso de los pastores del cantón de Santiago de Machaca, La Paz, Bolivia. En Pastoreo altoandino: Realidad, sacralidad y posibilidades, J. Flores y Y. Kobayashi (Eds.), pp. 111-134. MUSEF - Plural, La Paz.

NACHTIGALL, H., 1966. Indianische Fischer, Feldbauer und Viehzüchter. Reimer Verlag, Berlin.

NIELSEN, A., 2001. Ethnoarchaeological perpsectives on caravan trade in the South-Central Andes. En Ethnoarchaeology of Andean South America: Contributions to archaeological method and theory, L. Kuznar (Ed.), pp. 163-201. University of Michigan Press, Ann Arbor.

ORLOVE, B., 1981. Native Andean pastoralists: Traditional adaptations and recent changes. En Contemporary nomadic and pastoral peoples: Africa and Latin America, P. Salzman (Ed.), pp. 95-136. Studies in Third World Societies 17. Department of Anthropology, Williamsburg/ Virginia.

PALACIOS RIOS, F., 1987. Tecnología del pastoreo. En $L a$ tecnología en el mundo andino, H. Lechtman y A. M. Soldi (Eds.), pp. 217-232. UNAM, México, D.F.

PLATT, T., 1986. Mirrors and maize. The concept of yanantin among the Macha of Bolivia. En Anthropological History of Andean Polities, J. V. Murra, N. Wachtel y J. Revel (Eds.), pp. 228-259. Cambridge University Press, Cambridge.

RESCANIERE, A., 2001. La pareja y el mito. Estudios sobre las concepciones de la persona y de la pareja en los Andes. Fondo Editorial de la PUCP, Lima.

ROSEN, E. VON, 1957. Un mundo que se va. Exploraciones y aventuras entre las altas cumbres de la cordillera de los Andes. Fundación Miguel Lillo, Tucumán.

SALZMAN, C. y J. GALATY (Eds.), 1990. Nomads in a changing world. Instituto Universitario Orientale, Nápoles.

SARMIENTO, M., 1951. Folklore del altiplano de Jujuy. Boletín de la Asociación Tucumana de Folklore 15/16: 145-164.

TROLL, C., 1968. The cordilleras of the tropical Americas. Aspects of climatic, phytogeographical and agrarian ecology. En Geo-ecology of the mountainous regions of the tropical Americas, C. Troll (Ed.), pp. 15-56. Dümmlers, Bonn.

1980. Las culturas superiores andinas y el medio geográfico. Allpanchis Phuturinqa 14: 3-56.

VALDERRAMA, R. y C. ESCALANTE, 1998. Matrimonio en las comunidades quechuas andinas. En Gente de carne y hueso. Las tramas de parentesco en los Andes, D. Arnold (Ed.), pp. 291-322. CIASE - ILCA, La Paz. 
VICENTE, A. DE y A. DE VICENTE, 1942. Una escuela puneña. Pasión y aventura de dos maestros. El Ateneo, Buenos Aires.

WACHTEL, N., 1990. Le retour des ancêtres. Les Indiens Urus de Bolivie XXe - XVIe siècle. Essai d'histoire regressive. Gallimard, Paris.

WEBSTER, D., 1973. Native pastoralism in the South Andes. Ethnology 12: 115-133.

WEST, T., 1983. Family herds-individual owners. Livestock ritual and inheritance among the aymara of Bolivia. En
The keeping of animals: Adaptation and social relations in livestock producing communities, R. Berleant-Schiller y E. Shanklin (Eds.), pp. 93-106. Allanheld y Osmun, Totowa.

WINTERHALDER, B. y E. A. SMITH (Eds.) 1981. Huntergatherer foraging strategies. University of Chicago Press, Chicago.

YACOBACCIO, H., C. MADERO y M. MALMIERCA, 1998. Etnoarqueología de pastores surandinos. GZC, Buenos Aires. 